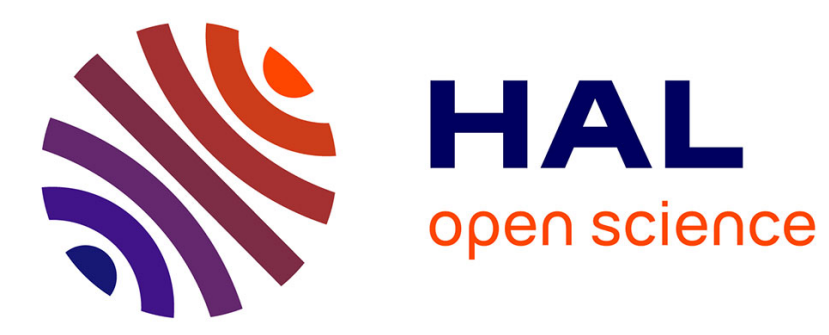

\title{
Two-point wavepacket modelling of jet noise
}

I. A Maia, Peter Jordan, A. V G Cavalieri, V. Jaunet

\section{To cite this version:}

I. A Maia, Peter Jordan, A. V G Cavalieri, V. Jaunet. Two-point wavepacket modelling of jet noise. Proceedings of the Royal Society A: Mathematical, Physical and Engineering Sciences, 2019, 475 (2227), pp.20190199. 10.1098/rspa.2019.0199 . hal-02348940

\section{HAL Id: hal-02348940 \\ https://hal.science/hal-02348940}

Submitted on 5 Nov 2019

HAL is a multi-disciplinary open access archive for the deposit and dissemination of scientific research documents, whether they are published or not. The documents may come from teaching and research institutions in France or abroad, or from public or private research centers.
L'archive ouverte pluridisciplinaire HAL, est destinée au dépôt et à la diffusion de documents scientifiques de niveau recherche, publiés ou non, émanant des établissements d'enseignement et de recherche français ou étrangers, des laboratoires publics ou privés. 


\section{PROCEEDINGS A}

rspa.royalsocietypublishing.org

Research

Article submitted to journal

\section{Subject Areas:}

Fluid mechanics, Aeroacoustics

\section{Keywords:}

Jet noise, wavepackets, kinematic modelling

\section{Author for correspondence:}

\section{A. Maia}

e-mail: igor.albuquerque.maia@univpoitiers.fr

\section{Two-point wavepacket modelling of jet noise}

\author{
I. A. Maia ${ }^{1}$, P. Jordan ${ }^{1}$, A. V. G. Cavalieri ${ }^{2}$ \\ and V. Jaunet ${ }^{1}$ \\ ${ }^{1}$ Institut PPRIME CNRS - Université de Potiers - \\ ENSMA, Poitiers, 86036, France \\ ${ }^{2}$ Divisão de Engenharia Aeronáutica, Instituto \\ Tecnológico de Aeronáutica, São José dos Campos, \\ 122228-900,Brazil
}

This paper is focused on the study of a kinematic wavepacket model for jet noise based on two-point statistics. The model contains physical parameters that define its structure in terms of wavenumber, envelope shape and coherence decay. These parameters, which are necessary to estimate the sound pressure levels radiated by the source, were educed from a large-eddy simulation database of a Mach 0.4, fully turbulent jet. The sound pressure levels predicted by the model were compared with acoustic data and the results show that when the parameters are carefully educed from the data, the soundpressure levels generated are in good agreement with experimentally measured values for low Strouhal numbers and polar angles. Furthermore, here we show that a correct representation of both coherence decay and wavepacket envelope shape are key aspects to an accurate sound prediction. A Spectral Proper Orthogonal Decomposition (SPOD) of the model source was also performed motivated by the search for a low-rank model capable of capturing the acoustic efficiency of the full source. It is shown that only a few SPOD modes are necessary to recover acousticallyimportant wavepacket traits.

\section{Introduction}

Jet noise is a challenging and compelling problem in fluid mechanics, because of its environmental and societal relevance but also because of disagreements that exist in the aeroacoustics community regarding its theoretical basis. Despite nearly seventy years of research, there persists a lack of consensus regarding the flow structures responsible for sound generation. The first mathematical treatment for the jet-noise problem was given by Lighthill

(C) The Authors. Published by the Royal Society under the terms of the Creative Commons Attribution License http://creativecommons.org/licenses/ by $/ 4.0 /$, which permits unrestricted use, provided the original author and source are credited. 
by means of his acoustic analogy. The main idea of the acoustic analogy is to rearrange the equations of motion as an inhomogeneous wave equation that separates the linear propagation of acoustic waves from a nonlinear inhomogeneous term composed of turbulent fluctuations that drive the sound field. In this framework, the turbulent field is replaced by an equivalent distribution of quadrupole sources whose statistical properties are related to those of the flow. Other acoustic analogies have since been proposed, for example, by Lilley [2] and Goldstein [3], that involve different source-propagator splits to deal with the effects of flow-acoustic interactions, but with the same central idea.

Because Lighthill's acoustic analogy is an exact rearrangement of the Navier-Stokes equation, it is possible to compute the sound field accurately if a sufficiently good description of the flow field is known. This has been strictly demonstrated for low Mach number and low frequency by Crow [4]. For higher Mach numbers, it has been demonstrated by means of direct numerical simulations by Freund [5] and Colonius and Freund [6] that accurate sound predictions are also possible if the source term is calculated in the close vicinity of the jet, so as to converge the convolution between source and Green's function. However, understanding and modelling the flow entities responsible for sound generation is a daunting task, as a correct description of the source terms in acoustic analogies depends on two-point, fourth-order statistics, finely described in a volume sufficient to cover all of the source region. Given this challenge, a considerable body of work has been undertaken to characterise flow parameters important for sound radiation and to build statistical source models. The structure of the cross-correlation tensor, which is at the core of the models, has frequently been analysed by hot-wire measurements, for example, by Davies et al. [7], Bradshaw et al. [8], Davies [9], Chu [10], who focused mainly on the characterisation of length and timescales; Harper-Bourne [11, 12] went further, determining the frequency-dependence of coherence and integral lengthscales at selected points in the mixing layer with a pair of hotwires; and Morris and Zaman [13] performed a similar analysis for positions both in the mixing layer and at the centerline of the jet. Two-point measurements have also been performed using LDV by Jordan and Gervais [14] and Kerhervé et al. [15] for limited regions in the jet, and the frequency-dependent character of turbulence statistics has also been explored. Other studies have used PIV to probe the structure of the cross-correlation tensor in $x-r$ and $r-\theta$ planes, as done for example by Ukeiley et al. [16], who evaluated the structure of linear and quadratic source components separately. Similar measurements, using PIV, have been performed by Seiner et al. [17] and Bridges and Wernet [18]. Jaunet et al. [19] performed time-resolved, stereo PIV in two synchronised crossflow planes with a view to studying two-point coherence of wavepackets and comparing them to those of the energy-containing, turbulent eddies. Different components of the cross-correlation tensor have also been analysed by Karabasov et al. [20] using a large-eddy simulation (LES) database.

The measurements of Harper-Bourne [11, 12] have been important in guiding noise-source modelling and in supporting acoustic prediction, as, for example, in the works of Self [21], Goldstein and Kharavan [22], Goldstein and Leib [23], Karabasov et al. [20] and Leib and Goldstein [24]. In these studies, model calculations are compared with acoustic data, and reasonable agreement is obtained for given frequency and polar-angle ranges.

While different source models are based on different descriptions of the two-point crosscorrelation function, one thing they all have in common is that they require knowledge of the variation of this function with position and frequency. In previous studies the cross-correlation function was generally obtained at a small selection of positions; given this constraint, simplifying assumptions are required. For example, length and timescales are assumed to be vary linearly with the ratio of kinetic energy by dissipation [22]; or the turbulence autocovariance tensor is assumed to be constant throughout the jet [24]; or the expansion coefficents. Moreover, again due to the lack of data, accurate sound prediction usually relies on the calibration of a number of empirical constants [21-26].

The modelling studies cited above consider sound sources based on a superposition of statistically-independent, uncorrelated eddies, a view aligned with the interpretation of the 
acoustic analogy given by Lightill. The parameters used in these models, such as convection velocity and length and timescales are those of the energy containing eddies, and are obtained from the integral length and timescales of turbulence. An alternative interpretation for the flow motions associated with sound generation came with the observation of wavepackets in turbulent jets by the experiments performed, for instance, by Mollo-Christensen [27, 28] and Crow and Champagne [29]. Wavepackets are organised, low-energy structures correlated over distances that far exceed the integral length scales of turbulence. The discovery of this underlying order and its connection with sound radiation motivated researchers to explore wavepacket-like source models. We can make a distinction between two kinds of wavepacket models: dynamic and kinematic [30]. Kinematic models are built based on experimental observations or statistical properties of a flow and are usually coupled with an acoustic analogy; they are inherently empirical and mask the details of the nonlinear fluid motions by which they are underpinned. A dynamic model based on the equations of motion is necessary to obtain the underlying flow motions. Hydrodynamic stability theory has been used as a theoretical framework that would provide a dynamic law governing the evolution of wavepackets in turbulent flows. Early examples of these modelling efforts can be found in the works of Michalke [31, 32], Crow and Champagne [29] and Crighton and Gaster [33]. Jordan and Colonius [34] made an extensive review of studies that have successfully used stability analysis, experiments and simulations (or a combination of these) to provide evidence for the existence of wavepackets in both the turbulent field [35-37] and the near-irrotational field [38-41]. It has also been demonstrated that the lowfrequency, far-field sound is consistent with an axially-extended wavepacket [31, 42-44] and that it can be decomposed, almost entirely, into only three azimuthal Fourier modes [43-46].

Modelling wavepackets using linear stability theory makes it possible to capture the evolution of their power-spectral density (PSD) up to the streamwise position at which they become neutrally stable [35]. However, further downstream there is considerable discrepancy between experiment and linear theory. For low Strouhal numbers $(S t<0.3)$ the agreement is also poor. Furthermore, linear dynamic models describe harmonic solutions, so they cannot describe the two-point statistics of a turbulent flow and this may explain the lack of success of these models in predicting correct pressure levels in the far-field. Acoustic extrapolation of wavepacket solutions of the Linearised Euler Equations (LEE) leads to a 30dB discrepancy in the far field [47]. In this spirit, a series of other papers have evoked the importance of coherence decay [41, 47-50] for acoustic efficiency. It is the statistical signature of the spatial desynchronisation of wavepackets due to turbulent forcing, a phenomenon that has been referred to as 'jitter' by Cavalieri et al. [51] and also modelled by Ffwocs-Williams and Kempton [52]. Cavalieri and Agarwal [48] showed that agreement in average phases and convection velocities obtained using linear models is not a sufficient condition to match the far pressure field of a sound source. A sufficient condition involves matching, in addition to amplitude and phase velocity, the two-point coherence of the source.

Kinematic models, as opposed to dynamic models based on stability theory, are useful in so far as they provide a framework that allows the salient sound-producing flow traits to be understood. Based on experiments, kinematic wavepacket source models have been proposed and their radiated sound field assessed, for example, by Crow [42], Crighton [53], Michalke [31] and Crighton and Huerre [54]. These studies proposed wavepacket models with similar shapes based on single-point measurements, and therefore did not consider coherence decay. More recently, using Crow's model [42], Cavalieri et al. [44] inferred the single-point wavepacket structure from the single-point statistics of the sound field and found a superdirective wavepacket consistent with the polar structure of the sound field for azimuthal Fourier modes $m=0,1$ and 2 and $0.2 \leqslant S t \leqslant 0.8$. The length of the wavepacket envelope was estimated between 6 and 8 jet diameters and a non-dimensional envelope length of $k_{h} L=6$ was found, where $k_{h}$ is the hydrodynamic wavenumber. The phase speed was taken from a local stability analysis. This model has since been widely used to study both free and installed jet noise [55-58]. Given that the importance of modelling two-point statistics was recognized early by Harper-Bourne [11, 12] 
and by Michalke [59], it is somewhat surprising that no wavepacket models featuring coherence decay were proposed earlier. It is only since the works of Reba et al. [60, 61, 62, 63] and Cavalieri and Agarwal [48] that wavepacket models featuring coherence decay have been proposed.

Now that is has become clear that the two-point coherence formulation comprises essential wavepacket source characteristics, the following question arises: to what extent are the sourcemodel characteristics identified by Cavalieri et al. [44] representative of actual wavepackets in the turbulent jet? As with all inverse-like source-identification approaches, which are ill-posed, one can always find a set of parameters that produces the correct sound field, but which may not be compatible with the real source structure.

In light of this, the objective and novelty of the work reported here is to use the two-point model of Cavalieri and Agarwal [48] combined with data from an LES simulation performed using the compressible flow solver 'Charles' [64], to identify and understand the key wavepacket parameters and to evaluate the acoustic radiation of the model, in terms of both directivity and acoustic efficiency. Whereas Cavalieri et al. [44] worked from the outside in, i.e inferring the source structure from the sound field, we here work from the inside out, building the source with information from the computed velocity field, the goal being to effect a quantitative calculation of the sound field using the two-point kinematic model whose parameters are this time extracted directly from the turbulent field. This approach follows the earlier ones by HarperBourne [11, 12], Goldstein and Kharavan [22], Goldstein and Leib [23], Karabasov et al. [20] and Leib and Goldstein [24], except that here we work with a wavepacket model source characterised by a small number of parameters, and we inform this using full-field flow data. The single and two-point acoustic predictions are compared with experiments conducted at the Bruit et Vent anechoic facility located in Poitiers, France. We focus the analysis on acoustic results at low polar angles to the jet axis for a single azimuthal mode, $m=0$, and we show that this approach considerably simplifies the modelling task; furthermore, the acoustic predictions of the model were found to agree with the data to within approximately $2 \mathrm{~dB}$ up to $S t=0.5$. Our methodology also builds on previous work by Kopiev and Chernyshev [65] and Kopiev and Chernyshev [66], who performed an azimuthal decomposition of the sound field and explored sound-source generation mechanisms of different azimuthal modes using the two-stage correlation model developed by Kopiev and Chernyshev [67]. Wavepacket models featuring coherence decay have also been used by Reba et al. $[60,63]$ to make acoustic predictions, although with a different methodology than ours; the authors used data from a near-field microphone array and performed an acoustic extrapolation of the near-field data to the acoustic field by solving the homogeneous wave equation. While their model worked well for supersonic jet conditions, for subsonic Mach numbers therir underpredicted the data by roughly $10 \mathrm{~dB}$ at low polar angles.

The kinematic modelling we propose is related to the dynamic models as follows. The shortcomings of linear dynamic models can be overcome by including an inhomogeneous, forcing term, associated with nonlinear interactions understood as the effect of turbulence forcing on wavepackets. A series of studies have explored forced, inhomogeneous models for the NavierStokes equations [68-72], addressing the problem in terms of an "input-output" analysis, where the input corresponds to the forcing and the output to the response modes of the flow, these being connected by the resolvent of the linearised Navier-Stokes equations. More recently, Semeraro et al. [73], Towne et al. [74] and Towne et al. [75] explored the link between resolvent modes and Spectral Proper Orthogonal Decomposition (SPOD) (a frequency domain form of Proper Orthogonal Decomposition) modes in turbulent jets. They show, consistent with the work of Farell and Ioannou [68], that when the linear system is forced with a spatiotemporally white stochastic field, the response modes of the resolvent operator are identical to the SPOD modes of the flow. This is not the case if the forcing is coloured and the difference between SPOD and resolvent modes may help shed light on the nature of the forcing.

This issue prompted us to perform SPOD of the kinematic source model with a view to searching for the acoustically-important degrees of freedom of wavepackets that might be used to construct a low-rank representation of the flow. We are interested in finding a reduced-rank 
description that suffices to provide an accurate description of the two-point structure that is relevant for sound radiation and that the dynamic models need to model correctly if they are to make accurate predictions of sound pressure levels.

Similarly to what was found by Breakey et al. [41] and Suzuki [76] in the near pressure field, we show here that a 1-mode representation of the Cross-Spectral Density (CSD) of the source is not sufficient to capture all of the acoustically-important features and that higher modes are important for sound radiation. But only only a small number of modes was necessary to recover the correct wavepacket parameters and sound pressure levels.

The remainder of the paper is organised as follows: In $\S 2$ we present the two-point kinematic source model. This is followed, in $\$ 3$, with the provision of information regarding the LES and acoustic databases. In $\S 4$ we describe the procedure used to educe the wavepacket parameters from the LES data. In $\S 5$ we compare the acoustic results of the model to experimental data and discuss the discrepancies and the role of the wavepacket parameters. In $\S 6$ we present the SPOD analysis and we discuss the possibilities of low-rank models for description of the acousticallyimportant wavepacket characteristics. Finally, conclusions ans hypotheses are discussed in $\S 7$.

\section{Kinematic Model}

\section{(a) Basic equations}

A generalised expression for an acoustic analogy can be written as

$$
\mathfrak{L}(p)=q(\mathbf{x}, t),
$$

where $\mathfrak{L}$ is a linear operator that depends on the acoustic analogy, $p$ is the pressure and $q(\mathbf{x}, t)$ is the source distribution.

The solution for the pressure, $p$, is given as

$$
p(\mathbf{x}, t)=-\int_{\mathscr{V}} \int_{-\infty}^{\infty} q(\mathbf{y}, \tau) G(\mathbf{x}, \mathbf{y}, t-\tau) \mathrm{d} \mathbf{y} \mathrm{d} \tau
$$

where $G(\mathbf{x}, \mathbf{y}, t-\tau)$ is the Green's function for the linear operator $\mathfrak{L}$. $\mathbf{x}$ is the observer's coordinates and $\mathbf{y}$ the source's coordinates.

Application of equation 2.2 involves integration of flow fluctuations, which are stationary random functions and hence are not square-integrable functions. A solution to overcome this issue is to work with the auto and cross-correlations, which are supposed to decay to zero for large separation distances and time delays [77]. The pressure autocorrelation is defined by

$$
\overline{p^{2}}(\mathbf{x}, \tau)=\lim _{T \rightarrow \infty} \frac{1}{2 T} \int_{-T}^{T} p(\mathbf{x}, t) p(\mathbf{x}, t+\tau) \mathrm{d} t,
$$

and that can be expressed, using equation 2.2 , as

$$
\begin{array}{r}
\overline{p^{2}}(\mathbf{x}, \tau)=\frac{1}{2 T} \int_{-T}^{T} \int_{-\infty}^{\infty} \int_{-\infty}^{\infty} \int_{\mathscr{V}} \int_{\mathscr{V}} G\left(\mathbf{x}, \mathbf{y}_{1}, t-\tau_{1}\right) G\left(\mathbf{x}, \mathbf{y}_{2}, t+\tau-\tau_{2}\right) \\
q\left(\mathbf{y}_{1}, \tau_{1}\right) q\left(\mathbf{y}_{2}, \tau_{2}\right) \mathrm{d} \mathbf{y}_{1} \mathrm{~d} \mathbf{y}_{2} \mathrm{~d} \tau_{1} \mathrm{~d} \tau_{2} \mathrm{~d} t .
\end{array}
$$

In the following, we follow the development of Goldstein and Leib [23]. We make three changes of variables $\boldsymbol{\eta}=\mathbf{y}_{2}-\mathbf{y}_{1}, \tau_{3}=\tau_{2}-\tau_{1}$ and $t_{1}=t-\tau_{1}$ and define $\mathscr{R}\left(\mathbf{y}_{1}, \boldsymbol{\eta}, \tau_{3}\right)$, a two-point time-delayed cross-correlation of the source based on flow quantities,

$$
\mathscr{R}\left(\mathbf{y}_{1}, \boldsymbol{\eta}, \tau_{3}\right)=\frac{1}{2 T} \int_{-T}^{T} q\left(\mathbf{y}_{1}, \tau_{1}\right) q\left(\mathbf{y}_{1}+\boldsymbol{\eta}, \tau_{1}+\tau_{3}\right) \mathrm{d} \tau_{1} .
$$

The expression for the pressure autocorrelation then becomes: 


$$
\overline{p^{2}}(\mathbf{x}, \tau)=\int_{-\infty}^{\infty} \int_{-\infty}^{\infty} \int_{\mathscr{V}} \int_{\mathscr{V}} G\left(\mathbf{x}, \mathbf{y}_{1}, t-\tau_{1}\right) G\left(\mathbf{x}, \mathbf{y}_{1}+\boldsymbol{\eta}, t+\tau-\tau_{2}\right) \mathscr{R}\left(\mathbf{y}_{1}, \boldsymbol{\eta}, \tau_{3}\right) \mathrm{d} \mathbf{y}_{1} \mathrm{~d} \boldsymbol{\eta} \mathrm{d} \tau_{3} \mathrm{~d} t
$$

We also define a modified Green's function, $G^{\prime}\left(\mathbf{x}, \mathbf{y}_{1}, \boldsymbol{\eta}, \tau-\tau_{3}\right)$, given by:

$$
G^{\prime}\left(\mathbf{x}, \mathbf{y}_{1}, \boldsymbol{\eta}, \tau-\tau_{3}\right)=\int_{-\infty}^{\infty} G\left(\mathbf{x}, \mathbf{y}_{1}, t_{1}\right) G\left(\mathbf{x}, \mathbf{y}_{1}+\boldsymbol{\eta}, t_{1}+\tau-\tau_{3}\right) \mathrm{d} t_{1} .
$$

Inserting equation 2.7 into equation 2.6 , we have

$$
\overline{p^{2}}(\mathbf{x}, \tau)=\int_{-\infty}^{\infty} \int_{\mathscr{V}} \int_{\mathscr{V}} G^{\prime}\left(\mathbf{x}, \mathbf{y}_{1}, \boldsymbol{\eta}, \tau-\tau_{3}\right) \mathscr{R}\left(\mathbf{y}_{1}, \boldsymbol{\eta}, \tau_{3}\right) \mathrm{d} \mathbf{y}_{1} \mathrm{~d} \boldsymbol{\eta} \mathrm{d} \tau_{3} .
$$

This equation can be equivalently expressed as:

$$
\overline{p^{2}}(\mathbf{x}, t)=\int_{-\infty}^{\infty} \int_{\mathscr{V}} \int_{\mathscr{V}} G^{\prime}(\mathbf{x}, \mathbf{y}, \boldsymbol{\eta}, t-\tau) \mathscr{R}(\mathbf{y}, \boldsymbol{\eta}, \tau) \mathrm{d} \mathbf{y} \mathrm{d} \boldsymbol{\eta} \mathrm{d} \tau .
$$

Equation 2.9 gives an expression for the pressure autocorrelation as a function of a Green's function and of the cross-correlation of the source, $\mathscr{R}$. The latter is a function of coordinate vector, $\mathbf{y}$, separation distance, $\boldsymbol{\eta}$, and time delay, $\tau$. Modelling this quantity is not a straightforward task, and this has obliged authors in the past to add free parameters to calibrate their models. The reason is that the modelling requires knowledge of the cross-correlation structure in all of the volume $\mathscr{V}$ where the source is defined; the information about how it varies with all of the components of $\mathbf{y}, \boldsymbol{\eta}$, and $\tau$ is usually not entirely available from experiments, or is limited to certain regions of the jet. This difficulty is true to all acoustic analogies. Here we wish to show that the modelling task is simplified by working in the frequency domain and concentrating on a single azimuthal mode and low polar angles to the jet axis. This is made possible by the homogeneity of the flow in the time and azimuthal directions.

The sound source model we consider is based on the Lighthill's acoustic analogy,

$$
\frac{1}{c_{0}} \frac{\partial^{2} p}{\partial t^{2}}-\nabla^{2} p=q_{i j}
$$

where $\mathbf{x}$ denotes spatial coordinates, $t$ is time, $p$ is the pressure, $c_{0}$ is the ambient speed of sound and $q$ is Lighthill's tensor, given by [31],

$$
q_{i j}=\frac{\partial^{2}}{\partial x_{i} \partial x_{j}}\left[\rho u_{i} u_{j}-\nu_{i j}\right]+\frac{1}{c_{0}^{2}} \frac{\partial^{2}}{\partial t^{2}}\left[p-c_{0}^{2} \rho\right],
$$

where $\rho$ is the density of the fluid, $u$ is the velocity, and $\nu_{i j}$ is the viscous term. Here we focus only on the $q_{11}$ term, which has been shown in previous studies to constitute an appropriate simplification for calculation of sound radiation to low polar angles [44, 53,55]. Moreover, we also use the conclusions of the studies of Freund [5], Bodony and Lele [78] and Colonius and Freund [6] to further simplify the source description. Freund [5] and Bodony and Lele [78] showed, by means of DNS and LES simulations, respectively, that at low Strouhal numbers $(S t<0.5)$ and polar angles, noise generation is dominated by the linear part of Lighthill's tensor in a Mach 0.9 jet; furthermore, they showed that over the same Strouhal-number and polar-angle range, the momentum term, $\rho u_{i} u_{j}$, is dominant over the entropic term, $\left[p-c_{0}^{2} \rho\right]$. As for the viscous term, Colonius and Freund [6] found it to be negligible to the sound field of a jet with a Reynolds number as low as 2000. In light of these results, we choose to drop the viscous and entropic terms and to only model the linearised part of the stress tensor. This compromises the performance of the model at higher Strouhal numbers and polar angles. However, our main objective here is to explore a simplified model with a view to providing insight into sound generation by wavepackets, hence the main message of this study is not affected by these simplifications. The Lightill stress tensor then reduces to 


$$
q_{11}=\frac{\partial^{2}}{\partial x^{2}}\left[2 \rho \bar{u}_{1} u_{1}^{\prime}\right]
$$

where $\bar{u}_{1}$ and $u_{1}^{\prime}$ are the streamwise mean and fluctuation velocities, respectively.

The solution to Lightill's acoustic analogy for the $m-t h$ azimuthal pressure component in frequency domain for an observer in the far field was given by Michalke [31],

$$
p_{m}(R, \theta, \omega)=\frac{i^{m} e^{-i k_{a} R} k_{a}^{2} \cos ^{2} \theta}{2 R} \iint Q_{m}(x, r, \omega) e^{i k_{a} x^{\prime} \cos \theta} J_{m}\left(k_{a} r \sin \theta\right) r \mathrm{~d} r \mathrm{~d} x,
$$

where the observer is in polar coordinates and the source in cylindrical coordinates. $k_{a}$ is the acoustic wavenumber, $J_{m}$ is the Bessel function of first kind and order $m$. $Q$ is the Fourier transform of the argument of the differential operator of $q_{11}$ and the double derivative was passed to the Green's function using the divergence theorem, as demonstrated by Goldstein [79].

The power spectral density of the pressure, which is the Fourier transform of the pressure autocorrelation defined in equation 2.9 , is then given by

$$
\begin{gathered}
\left\langle p_{m}(R, \theta, \omega) p_{m}^{*}(R, \theta, \omega)\right\rangle=(-1)^{m} i^{2 m} \frac{k_{a}^{4} \cos ^{4} \theta}{4 R^{2}} \\
\iiint \int\left\langle Q_{m}\left(x_{1}, r_{1}, \omega\right) Q_{m}^{*}\left(x_{2}, r_{2}, \omega\right)\right\rangle e^{i k_{a} \cos \theta\left(x_{1}-x_{2}\right)} J_{m}\left(k_{a} r_{1} \sin \theta\right) J_{m}\left(k_{a} r_{2} \sin \theta\right) r_{1} r_{2} \mathrm{~d} r_{1} \mathrm{~d} r_{2} \mathrm{~d} x_{1} \mathrm{~d} x_{2},
\end{gathered}
$$

where the symbols $\langle$.$\rangle denote expected value. The quantity \left\langle Q_{m}\left(x_{1}, r_{1}, \omega\right) Q_{m}^{*}\left(x_{2}, r_{2}, \omega\right)\right\rangle$ is the cross-spectral density of the source and is equivalent to the Fourier transform of the cross-correlation, $\mathscr{R}$.

In this work we are interested in the $m=0$ axisymmetric mode. For small values of the argument of the Bessel function, $k_{a} r \sin \theta=2 \pi S t M r / D \sin \theta \ll 1, J_{0}$ is approximately 1 , and the Bessel function term can be neglected. Table 1 shows values of the argument of the Bessel function for the range of Strouhal numbers, radial positions and polar angles we consider in this work. We can see that the accuracy of the results at high angles, which is already limited by the linearisation of the source, is clearly affected by neglecting the Bessel function. For $S t=0.5$ and higher, this simplification begins to affect also low polar angles. More considerations about the limits to this simplification and how it affects the present modelling strategy are made in section $\S 5$.

Table 1: Values of $J_{0}(2 \pi S t M r / D \sin \theta)$

\begin{tabular}{c|cc|cc|cc}
\hline & \multicolumn{2}{|c|}{$S t=0.3$} & \multicolumn{2}{c|}{$S t=0.5$} & \multicolumn{2}{c}{$S t=0.7$} \\
& $\theta=20^{\circ}$ & $\theta=90^{\circ}$ & $\theta=20^{\circ}$ & $\theta=90^{\circ}$ & $\theta=20^{\circ}$ & $\theta=90^{\circ}$ \\
\hline$r / D=0.2$ & 0.99 & 0.99 & 0.99 & 0.98 & 0.99 & 0.97 \\
$r / D=1$ & 0.98 & 0.86 & 0.95 & 0.64 & 0.91 & 0.36 \\
$r / D=2$ & 0.94 & 0.51 & 0.82 & -0.06 & 0.67 & -0.38 \\
\hline
\end{tabular}

The expression for the axisymmetric component of the PSD then becomes:

$$
\begin{gathered}
\left\langle p_{0}(R, \theta, \omega) p_{0}^{*}(R, \theta, \omega)\right\rangle=\frac{k_{a}^{4} \cos ^{4} \theta}{4 R^{2}} \\
\iiint \int\left\langle Q_{0}\left(x_{1}, r_{1}, \omega\right) Q_{0}^{*}\left(x_{2}, r_{2}, \omega\right)\right\rangle e^{i k_{a} \cos \theta\left(x_{1}-x_{2}\right)} r_{1} r_{2} \mathrm{~d} r_{1} \mathrm{~d} r_{2} \mathrm{~d} x_{1} \mathrm{~d} x_{2} .
\end{gathered}
$$

Our approach then consists in using a line-source function, $S\left(x_{1}, x_{2}, \omega\right)$, to model the radiallyintegrated source, 


$$
\int\left\langle Q\left(x_{1}, r_{1}, \omega\right) Q^{*}\left(x_{2}, r_{2}, \omega\right)\right\rangle r_{1} r_{2} \mathrm{~d} r_{1} \mathrm{~d} r_{2} \approx S\left(x_{1}, x_{2}, \omega\right) .
$$

The subscripts referring to azimuthal mode have been dropped for convenience, but hereafter, all of the flow and acoustic information discussed refers to the axisymmetric mode.

We can see the modelling advantage of only taking into account the $m=0$ azimuthal mode and concentrating at low polar angles and Strouhal numbers: instead of having to model the full cross-spectral density (or the cross-correlation, its counterpart in time domain) and how it varies with source position and separation distance (equation 2.9), here we are left with the much simpler task of modelling the two-point statistics for a pair of points $\left(x_{1}, x_{2}\right)$ in the streamwise direction. It is worth emphasising that this approach is only possible due to the significant amount of information provided by the LES, which contains the radial structure of the source and thus allows the radial integration of equation 2.16.

The present modelling methodology is different from past studies that used a wavepacket linesource approach $[44,51,58,80]$ to the extent that we do not chose a specific radial position from which to extract flow information and build the model; instead, we model the radially-integrated Lightill stress tensor, so that equation 2.16 uses all the information available from the numerical database and yields a line-source model that contains information about the radial structure of the source.

\section{(b) Wavepacket source model}

Here we use modified versions of the model of Cavalieri and Agarwal [48] for the source, which comprises a line-source wavepacket of CSD, $S\left(x_{1}, x_{2}, \omega\right)$, with modulated amplitude and coherence. The coherence and amplitude envelopes were modified so as to better represent the flow information extracted from the simulation. For the coherence envelope (last term on the right-hand side of equation 2.17) we used a model proposed by Jordan et al. [81] and O'Hara et al. [82], which consists in the convolution of Gaussian and exponential functions, as opposed of the Gaussian used by Cavalieri and Agarwal [48]. The reason for this, as discussed in §4, is that the convolution allows an accurate description of coherence at small separation distances. Moreover, two envelope functions have been used: a standard Gaussian used in pas studies [44, 48, 53, 54] and and a modified Gaussian that has an asymmetric decay rate. Asymmetric wavepacket models have also been explored by Papamouschou [83] and Koenig et al. [84]. The expression for the CSD with a Gaussian envelope is given as

$$
\begin{aligned}
& S\left(x_{1}, x_{2}, \omega\right)=A(\omega) \exp \left[i k_{h}(\omega)\left(x_{1}-x_{2}\right)\right] \\
& \exp \left[-\frac{\left(x_{1}-x_{0}(\omega)\right)^{2}}{L(\omega)^{2}}-\frac{\left(x_{2}-x_{0}(\omega)\right)^{2}}{L(\omega)^{2}}\right] \gamma^{2}\left(x_{1}, x_{2}, \omega\right)
\end{aligned}
$$

where $k_{h}(\omega)$ is the hydrodynamic wavenumber, $L(\omega)$ is the characteristic wavepacket length, and $\gamma^{2}\left(x_{1}, x_{2}, \omega\right)$ is the coherence function. $A(\omega)$ is a term that sets the amplitude of the source. It is taken as the maximum amplitude of the integrated source tensor, which occurs at the streamwise position corresponding to the center of the wavepacket, $x_{0}(\omega)$, which is itself a function of frequency.

The coherence function, $\gamma^{2}\left(x_{1}, x_{2}, \omega\right)$, is given as

$$
\gamma^{2}\left(x_{1}, x_{2}, \omega\right)=\frac{1}{2 L_{c_{1}}} \int_{-\infty}^{\infty} e^{-\frac{\left|x_{1}-x_{2}-\eta\right|}{L_{c_{1}}}} e^{-\frac{\left(x_{1}-x_{2}\right)^{2}}{L_{c_{2}}{ }^{2}}} \mathrm{~d} \eta,
$$

where $L_{c_{1}}$ and $L_{c_{2}}$ are the lengthscales associated with the exponential and Gaussian functions, respectively and are function of $\omega$.

A Gaussian function provides a good approximation for the amplification part of the amplitude shapes (this is shown in section $\S 4$, figure 4). However, the decaying part is asymmetric 
and a Gaussian is not able to accurately represent the wavepacket envelope. Thus, an asymmetric Gaussian envelope has also been used to fit the envelopes, given by the function

$$
f(x)=\frac{1}{L_{a s}} e^{x^{2} / L_{a s}^{2}} \operatorname{erfc}\left[\frac{\alpha x}{\sqrt{2} L_{a s}}\right],
$$

where $L_{a s}$ is a characteristic length of the asymmetric envelope, $\alpha$ is a parameter controlling the decay part of the function and erfc is the complementary error function. The CSD with the asymmetric envelope becomes

$$
\begin{gathered}
S\left(x_{1}, x_{2}, \omega\right)=A(\omega) \frac{1}{L_{a s}(\omega)^{2}} \exp \left[i k_{h}(\omega)\left(x_{1}-x_{2}\right)\right] \exp \left[-\frac{\left(x_{1}-x_{0}(\omega)\right)^{2}}{L_{a s}(\omega)^{2}}-\frac{\left(x_{2}-x_{0}(\omega)\right)^{2}}{L_{a s}(\omega)^{2}}\right] \\
\operatorname{erfc}\left[\frac{\alpha(\omega)\left(x_{1}-x_{0}(\omega)\right)}{\sqrt{2} L_{a s}(\omega)}\right] \operatorname{erfc}\left[\frac{\alpha(\omega)\left(x_{2}-x_{0}(\omega)\right)}{\sqrt{2} L_{a s}(\omega)}\right] \gamma^{2}\left(x_{1}, x_{2}, \omega\right) .
\end{gathered}
$$

Both models are based on two-point statistics of the flow, since these take into account coherence decay. By making $L_{c_{1,2}} \rightarrow \infty$, one eliminates the two-point dependence and obtains a model based on single-point statistics. The parameters highlighted in equations 2.17 and 2.20 in red may then be educed from the simulation database after performing the integrals in the radial direction.

Therefore, using equations 2.17 or 2.20 once the parameters have been educed, one can evaluate the far-field radiation of the model source through the following equation:

$$
p(R, \theta, \omega) p^{*}(R, \theta, \omega)=\frac{k_{a}^{4} \cos ^{4} \theta}{4 R^{2}} \iint S\left(x_{1}, x_{2}, \omega\right) e^{i k_{a} \cos \theta\left(x_{1}-x_{2}\right)} \mathrm{d} x_{1} \mathrm{~d} x_{2} .
$$

It is worth emphasising that this model is contained in the more general source description given by equation 2.9, and explored, for example, by Kharavan et al. [26], Tam and Auriault [25], Self [21], Goldstein and Kharavan [22], Goldstein and Leib [23] and Leib and Goldstein [24], Kopiev and Chernyshev [65]. Wavepackets, like the source models proposed in those studies, are also of quadrupolar nature, but are characterised by longer space scales. By focusing on these specific flow entities, given their now demonstrated importance for sound radiation, we obtain a simplified line-source description that requires a relatively small number of parameters.

\section{Databases}

A large-eddy simulation of an isothermal $M a=0.4$ turbulent jet has been performed using the compressible solver 'Charles' [85]. The nozzle geometry and flow parameters reproduce the experimental setup of the Bruit et Vent noise facility of the PPRIME institute, located in Poitiers, France, and studied in previous studies [19, 35, 44]. The Reynolds number based on the jet diameter is $4.6 \times 10^{5}$, and synthetic-turbulence is used in the simulation to mimic the effect of the boundary layer trip present in the experiment at an streamwise distance of $x / D=2.5$ upstream of the jet exit. The simulation has been run for a total time of 2000 acoustic time units, where the acoustic time is defined as $t c_{0} / D$. The unstructured grid was interpolated to a cylindrical grid that covers a volume extending from $0 \leqslant x / D \leqslant 30,0 \leqslant r / D \leqslant 6,0 \leqslant \Phi \leqslant 2 \pi$. Schmidt et al. [85] used this same database to perform spectral proper orthogonal decomposition and resolvent analysis with a view to study the low-rank behaviour of turbulent jets. More details about the numerical method and meshing strategy can be found in A. et al. [46].

The acoustic measurements were performed with an azimuthal ring array of diameter $14.3 \mathrm{D}$ that contained 18 microphones. It is the same array previously used by Piantanida et al. [56]. The ring was displaced in the axial position in the range $0 D \leq x \leq 39 D$ so as to vary the polar angle, covering a cylindrical surface. This configuration allowed the decomposition of the pressure signal into Fourier modes up to $m=9$. A schematic of the acoustic experiment is shown in figure 


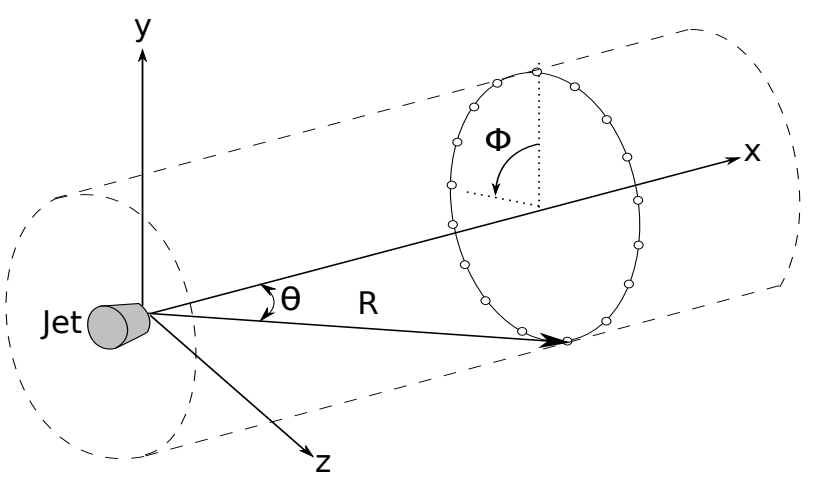

Figure 1: Schematic of microphone array.

1. Both flow and acoustic data have been Fourier transformed in the azimuthal direction in order to extract the $m=0$, axisymmetric mode.

Figure 2 compares the LES with experimental data for the same jet: flow statistics are compared with PIV data from Jaunet et al. [19] and sound pressure levels of the full sound field as a function of polar angle are compared with measurements made using the experimental setup described above. The sound field is computed from the LES by means of a permeable formulation of the Ffowcs Williams \& Hawking formulation in frequency domain [46, 64]. Mean flow and rms profiles are in good agreement with the PIV data. The experimental data seems to underpredict the rms levels of the LES near the nozzle, which can be explained by the low level of the magnification factor of the PIV at this position, as discussed by Jaunet et al. [19]. Directivities computed from the simulation are also in good agreement with experimental data.

\section{Parameter Eduction}

In what follows we describe the procedure adopted in order to educe the parameters necessary to build the CSD models given by equations 2.17 and 2.20 from the LES data. The optimal parameters were selected using the MATLAB ${ }^{\circledR}$ fminsearch routine, a nonlinear least-squares algorithm in which the error between data and model was minimised using the Nelder-Mead simplex method. The data consists of the full source CSD, $\left\langle Q\left(x_{1}, r_{1}, \omega\right) Q^{*}\left(x_{2}, r_{2}, \omega\right)\right\rangle$, dependent on two axial coordinates, two radial coordinates and frequency. Prior to the eduction, the data is radially integrated twice and the model is created based on the line-source that results from this operation, given by equation 2.16. Thus, all of the parameters have been educed for the integrated source, and the information regarding the radial structure of the source is, so to speak, contained in these and in the amplitude term. Since all the radial structure of the source available is taken into account, it is not necessary to pick a specific radial position at which to educe the parameters, unlike other studies that use a line-source approach $[44,51,58,80]$.

Figure 3 shows the real part of the radially-integrated CSD, $S\left(x_{1}, x_{2}, \omega\right)$, issued from the LES for $0.3 \leqslant S t \leqslant 0.7$ with the reference point at peak the wavepacket, $x_{0}$. The wave-like character of the source term of Lighthill's acoustic analogy is evident, which motivates the modelling efforts of the present study.

\section{(a) Wavepacket characteristic length, $L$}

The wavepacket characteristic length, $L$, was extracted by fitting the PSD of the velocity fluctuation signal with an envelope function. Two envelopes were used: one with a Gaussian form, which corresponds to the model of Cavalieri and Agarwal [48] and a second one with an asymmetric Gaussian form, which accounts for different growth and decay rates. The Gaussian envelope may be obtained by making $x_{2}=x_{1}$ in equation 2.17 . 

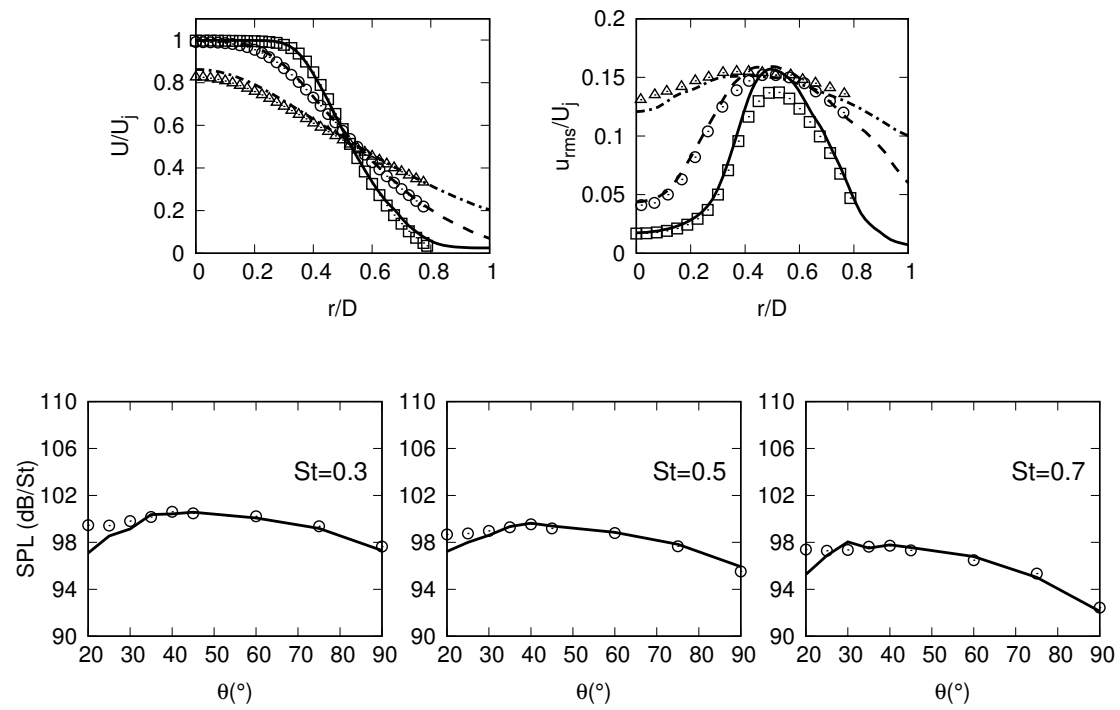

Figure 2: Top row: mean flow (left) and rms (right) radial profiles obtained from PIV (points) and LES (lines). Profiles at three streamwise stations are shown: $x / D=2.5$ (solid line and squares); $x / D=4.5$ (dashed-line and circles); $x / D=8$ (dash-dotted-line and triangles). Bottom row: directivity plots for three Strouhal numbers with observer in the cylindrical surface delimited by the microphone array (figure 1). Circles: experimental data; solid line: LES far field prediction computed using the Ffowcs Williams \& Hawkings equation.

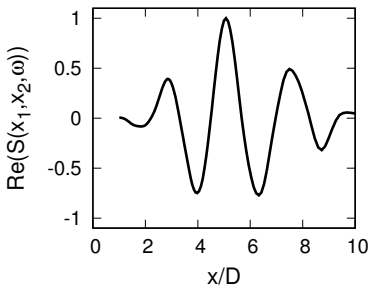

(a) $\mathrm{St}=0.3$

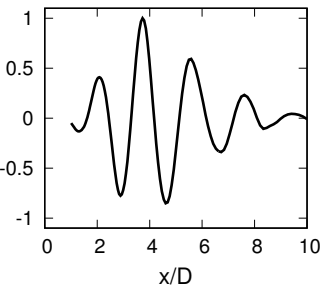

(b) $\mathrm{St}=0.4$

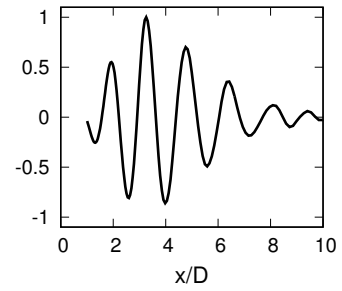

(c) $\mathrm{St}=0.5$

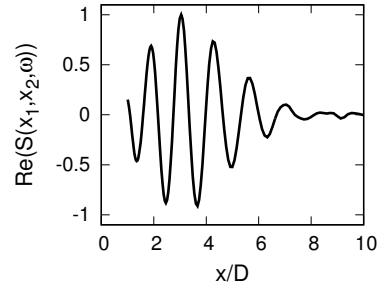

(d) $\mathrm{St}=0.6$

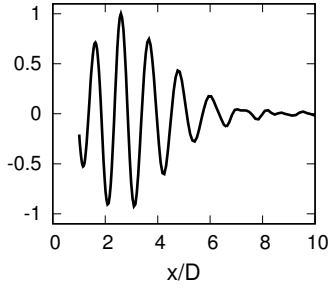

(e) $\mathrm{St}=0.7$

Figure 3: Radially-integrated CSD issued from the LES for different Strouhal numbers. Reference point is at $x_{0}(\omega)$. Levels have been normalised by the maximum value at each Strouhal number.

$$
S_{\text {sym }}\left(x_{1}, \omega\right)=A(\omega) \exp \left[-\frac{2\left(x_{1}-x_{0}(\omega)\right)^{2}}{L(\omega)^{2}}\right]
$$




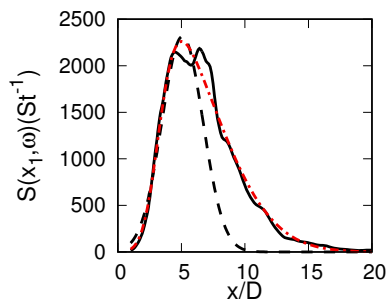

(a) $\mathrm{St}=0.3$

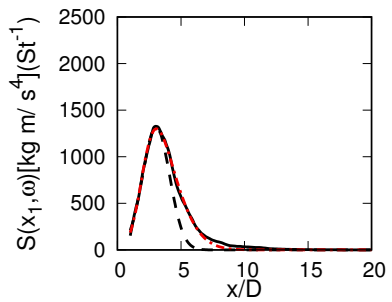

(d) $\mathrm{St}=0.6$

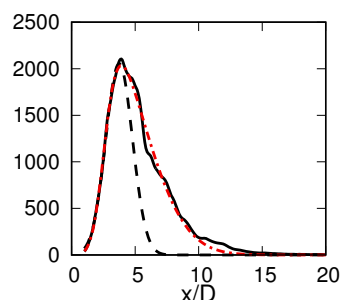

(b) $\mathrm{St}=0.4$

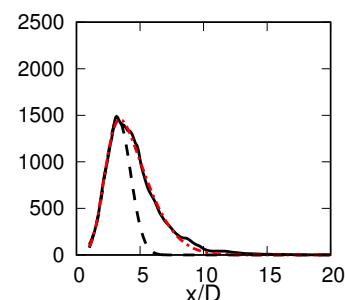

(c) $\mathrm{St}=0.5$

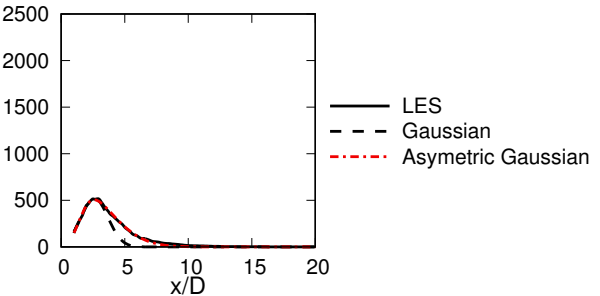

(e) $\mathrm{St}=0.7$

Figure 4: PSD of the source and fitted Gaussian and asymmetric envelopes.

where the amplitude term is the direct result of the double radial integration performed with equation 2.16. This function provides a reasonable fit of radially-integrated power spectral densities for the growth part of the wavepacket; however, downstream of the peak the agreement becomes poorer, and the Gaussian fit does not represent the data correctly. Hence, here we propose a modified Gaussian function that takes into account the asymmetry of the wavepacket. This function can be obtained by setting $x_{2}=x_{1}$ in equation 2.20,

$$
S_{a s y m}\left(x_{1}, \omega\right)=A(\omega) \frac{1}{L_{a s}(\omega)^{2}} \exp \left[-\frac{2\left(x_{1}-x_{0}(\omega)\right)^{2}}{L_{a s}(\omega)^{2}}\right] \operatorname{erfc}^{2}\left[\frac{\alpha\left(x_{1}-x_{0}(\omega)\right)}{\sqrt{2} L_{a s}(\omega)}\right] .
$$

One can observe, in Figure 4, that the asymmetric envelope provides a better match for the data in the decaying part on the envelope, where the Gaussian envelope significantly underpredicts the data. The acoustic radiation of the wavepacket model using both envelopes will be discussed in $\S 5$.

\section{(b) Hydrodynamic Wavenumber, $k_{h}$}

The hydrodynamic wavenumber and the phase speed are related through

$$
U_{c}\left(x_{1}, \omega\right)=\frac{\omega}{k_{h}}=\omega\left(\frac{\partial \phi}{\partial x_{2}}\right)^{-1}
$$

where $\phi\left(x_{1}, x_{2}\right)$ is the phase shift, obtained as the argument of the CSD. This expression gives the phase speed for reference point $x_{1}$ as a function of the rate of change of $\phi$ with the separation distance from two-points. We found this rate of change to be approximately constant in the LES data, so that the phase speed was educed from the simulation database by fitting the phase with a linear regression. Figure 5 shows the space-frequency variation of the phase speed. One can observe that the phase speed increases with increasing streamwise position and converges to a value of approximately $U_{c} / U_{j}=0.77$. Harper-Bourne [12] and Morris and Zaman [13] have used a power-law of the form $U_{c} / U_{j}=a \ln S t+b$ to model the variation of the phase speed with Strouhal number. Here we use the same function to take into account the variation of phase speed with reference position, $x_{1}$. The fit can also be seen in figure 5 . 


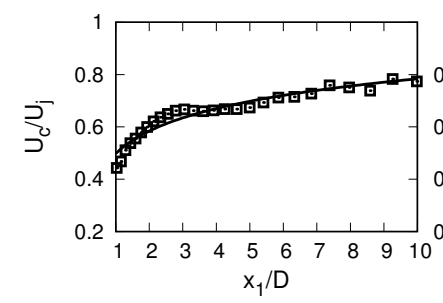

(a) $\mathrm{St}=0.3$

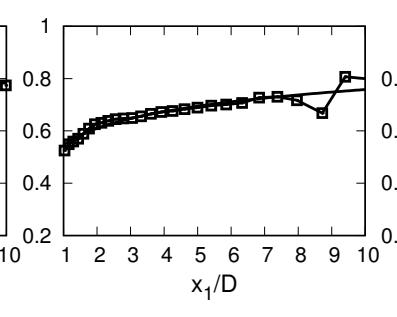

(b) $\mathrm{St}=0.4$

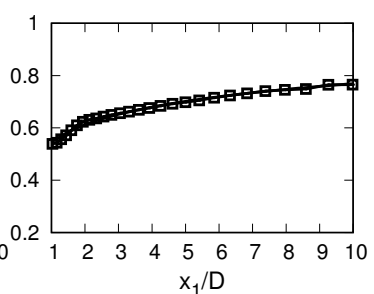

(c) $\mathrm{St}=0.5$

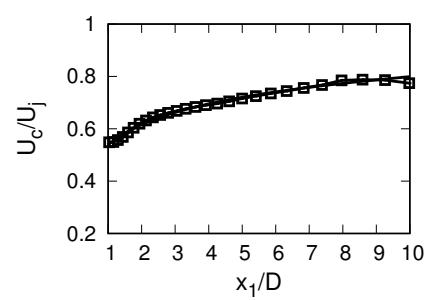

(d) $\mathrm{St}=0.6$

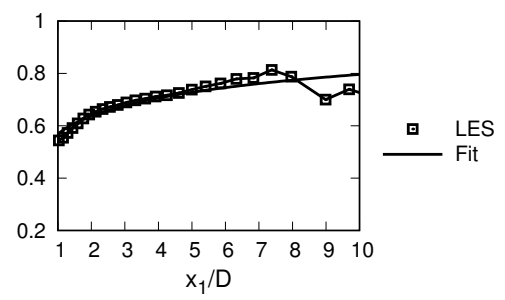

(e) $\mathrm{St}=0.7$

Figure 5: Phase velocity as a function of streamwise reference point, $x_{1}$, for different Strouhal numbers and power-law fit.

\section{(c) Coherence Lengths, $L_{c_{1}}, L_{c_{2}}$}

The magnitude-squared coherence of the radially-integrated source term $S\left(x_{1}, x_{2}, \omega\right)$, is given as,

$$
\gamma^{2}\left(x_{1}, x_{2}, \omega\right)=\frac{\left|\left\langle S\left(x_{1}, x_{2}, \omega\right)\right\rangle\right|^{2}}{\left\langle\left|S\left(x_{1}, \omega\right)\right|^{2}\right\rangle\left\langle\left|S\left(x_{2}, \omega\right)\right|^{2}\right\rangle},
$$

where $S\left(x_{1}, \omega\right)$ and $S\left(x_{2}, \omega\right)$ are the PSDs at points $x_{1}$ and $x_{2}$, respectively.

Jaunet et al. [19] used an exponential function to fit coherence decay for a $M a=0.4$; however, this function does no provide a correct description of coherence for values of separation distance $\Delta x \rightarrow 0$. Jordan et al. [81] and O'Hara et al. [82] have proposed, for the two-point correlation, a function given by the convolution of an exponential and a Gaussian. This function has the interesting property of keeping the decay rate of an exponential whilst providing a zero slope at $\Delta x=0$. In this work we use the function of Jordan et al. [81], given by equation 2.18, to fit the coherence of the LES data and obtain the two parameters that control the decay rate of the curve, $L_{c_{1}}$ and $L_{c_{2}}$. Figure 6 shows the magnitude-squared coherence as a function of streamwise separation distance for various Strouhal numbers and the fitted function. The reference position was set is the wavepacket peak, $x_{0}$. The zero slope at $\Delta x=0$ can clearly be observed in the data, whence the use of the convolution function. Tam and Auriault [25] and Kopiev and Chernyshev [86] have shown that a sharp behaviour of the correlation function near $\Delta x=0$ is important for a correct modelling of the broad-band spectra typical of high polar angles. Since this feature is not present in the $T_{11}$ term of Lighthill's tensor, here we argue that it is somewhat less important for sound radiation at low polar angles to the jet axis.

The decay rate of coherence has been found to vary considerably with reference position, $x_{1}$. Figure 7 shows the variation of $L_{c_{1}}$, the parameter that controls the global decay rate of coherence, as a function of $x_{1}$. This spatial dependence has been taken into account by fitting the coherence length with high order polynomials. $L_{c_{2}}$, which corrects the slope of coherence at small separation distances, has been found to be less sensitive to streamwise coordinate. The 


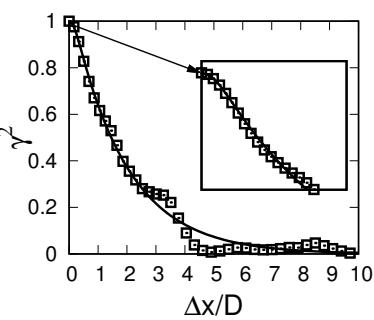

(a) $\mathrm{St}=0.3$

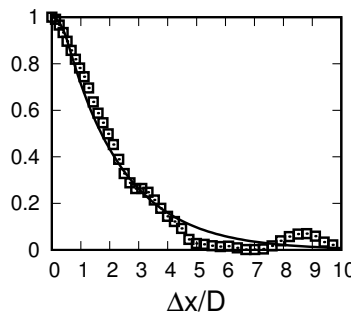

(b) $\mathrm{St}=0.4$

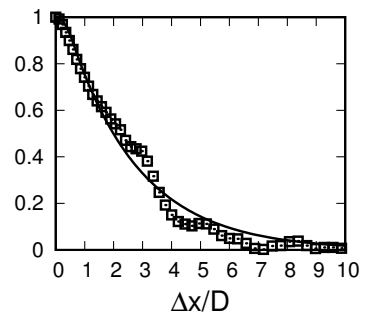

(c) $\mathrm{St}=0.5$

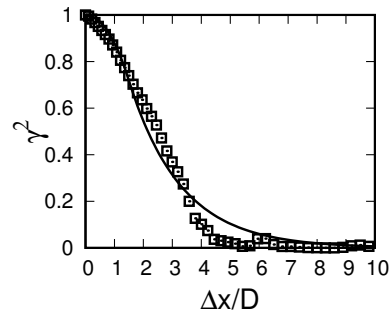

(d) $\mathrm{St}=0.6$

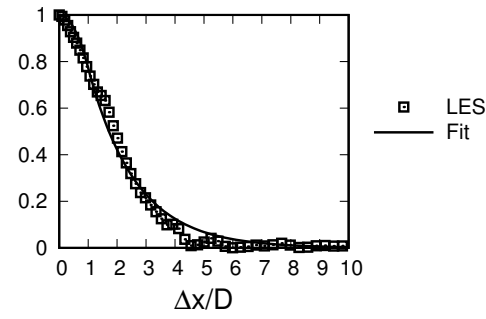

(e) $\mathrm{St}=0.7$

Figure 6: Magnitude-squared coherence of the LES data and fitted curves.

search for a general scaling for coherence lengths and an expression for their space-frequency dependence is something that will be considered in future work.

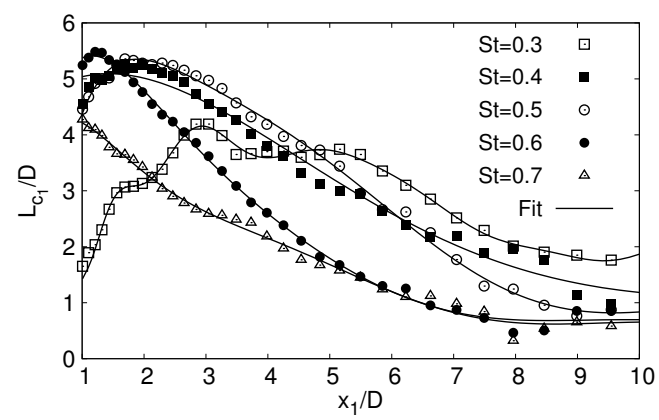

Figure 7: $L_{c_{1}}$ as a function of reference point for and polynomial fitting for various Strouhal numbers.

\section{(d) Comparison of educed parameters with previous wavepacket models}

Cavalieri et al. [44] used a Gaussian wavepacket source model based on single-point statistics of the sound field; coherence decay was thus not accounted for. As mentioned in the introduction, because of the ill-posed nature of this inverse problem, one may find more than one set of parameters that produce a source consistent with sound radiation from experimental results. These source parameters may not correspond to those of the jet however; the fact that the singlepoint model lacks coherence decay means that its effect is, so to speak, 'hidden' in the set of parameters found such that the correct sound field is obtained. Indeed, the value found by Cavalieri et al. [44] for the source characteristic length $L / D$ is underestimated with respect to 
the values educed from the LES data, as shown in table 2; since that work did not consider coherence decay in the source, it is possible that the lower value of $L / D$ in Cavalieri et al. [44] compensates for the effect of the coherence lengthscale in an effective length (such as the modified length $L_{m}$ in Cavalieri and Agarwal [48]). Also, the phase speeds identified in the LES data are significantly lower than that found from the stability analysis in that work, which was based on a mean velocity profile in the near-nozzle region, where, as can be seen in figure 5 , the phase speeds are considerably higher than those observed in most other regions of the jet. In what follows, we compare the single-point 'inverse' model of Cavalieri et al. [44] with the 'direct' two-point model considered in this work at $S t=0.2$.

Table 2: Wavepacket parameters at $S t=0.2$, educed at the wavepacket peak, $x_{0}$.

\begin{tabular}{cccc|cccc}
\hline \multicolumn{4}{c|}{ Present Study } & \multicolumn{4}{c}{ Cavalieri et al. [44] } \\
$k_{h} L$ & $L / D$ & $U_{c} / U_{j}$ & $L_{c_{1}} / D$ & $k_{h} L$ & $L / D$ & $U_{c} / U_{j}$ & $L_{c_{1}} / D$ \\
\hline 8.97 & 4.52 & 0.68 & 2.49 & 6.50 & 3.10 & $0.6,0.97$ & - \\
\hline
\end{tabular}

\section{Acoustic radiation of single- and two-point source models}

Here we compare the acoustic fields generated by single and two-point wavepacket models through equation 2.21, where the single-point model is computed by setting $L_{c_{1,2}}=\infty$ in equation 2.17, considering the model with a Gaussian envelope. Figure 8 shows the SPL in dB/St generated by both models, using Gaussian amplitude envelopes, compared with experimental data for the $m=0$ azimuthal mode at Strouhal numbers $S t=0.3-0.7$. Despite the implications of only modelling the linear part of the Ligthill tensor at high polar angles, as evoked in section $\S 2$, we show the results up to $90^{\circ}$. It is clear that, if coherence decay is neglected, as is the case for the single-point model, the SPL generated significantly underpredicts the experimental data. Other studies based on dynamic wavepacket modelling have come to a similar conclusion $[41,47,49,50]$. This further illustrates that the effect of coherence decay was somewhat hidden in, or compensated for by, the single-point parameters estimated from the acoustic field by Cavalieri et al. [44]. It must be emphasised that the acoustic data is measured with the observer on the cylindrical surface delimited by the microphone array. This does not correspond to a true polar reference frame, since the distance between source and observer decreases with increasing polar angle; for this reason the SPL decay rate with polar angle is smaller than that observed by [44].

In order to understand the effect of coherence on the source structure and sound radiation efficiency, it is interesting to look at the CSD in wavenumber space. Figure 9 shows a comparison of the spatial Fourier transform of the CSD of a source with unit coherence and another with coherence decay at $S t=0.3 . k_{x_{1}}$ and $k_{x_{2}}$ are wavenumbers corresponding to the $x_{1}$ and $x_{2}$ directions, respectively. From equation 2.21, which can be recognised as a double spatial Fourier transform, we realise that only the wavenumbers that are in the range $\left|k_{x_{1}}\right| \leq k_{a}$ and $\left|k_{x_{2}}\right| \leq k_{a}$ contribute to sound radiation. From the relation of wavenumber and phase speed given by equation 4.3 , we have,

$$
\begin{aligned}
& U_{c_{1}}>c, \\
& U_{c_{2}}>c
\end{aligned}
$$

where $c$ is the sound speed and $U_{c_{1}}$ and $U_{c_{2}}$ are the phase speeds associated with wavenumbers $k_{x_{1}}$ and $k_{x_{2}}$, respectively. This shows that only wavenumbers with supersonic associated phase speeds contribute to far-field radiation.

These conditions can also be written as 


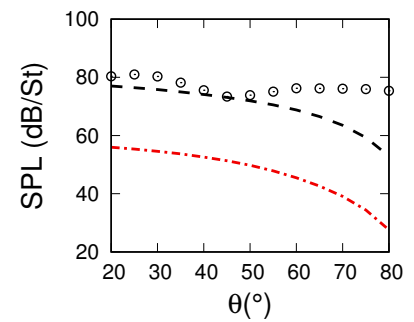

(a) $\mathrm{St}=0.3$

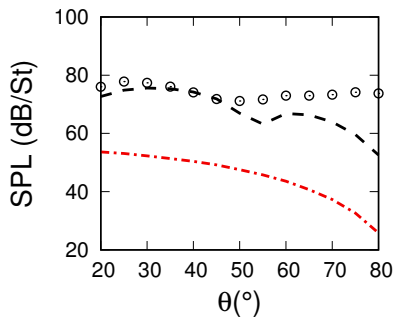

(d) $\mathrm{St}=0.6$

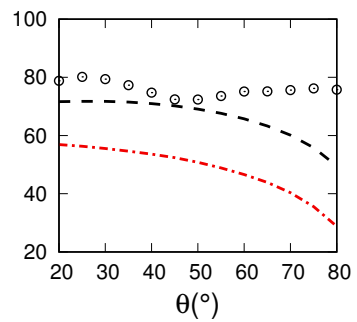

(b) $\mathrm{St}=0.4$

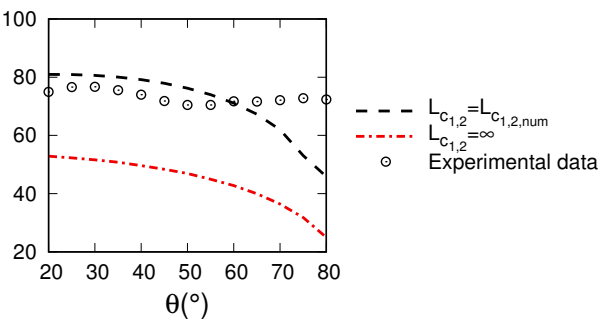

(e) $\mathrm{St}=0.7$

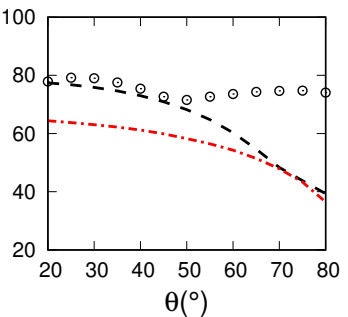

(c) $\mathrm{St}=0.5$

Figure 8: Comparison between sound radiated by single- and two-point wavepacket models and experimental data. The models have a Gaussian amplitude envelope.

$$
\begin{aligned}
& \frac{\left|k_{x_{1}}\right|}{k_{h}}<M_{c}, \\
& \frac{\left|k_{x_{2}}\right|}{k_{h}}<M_{c},
\end{aligned}
$$

as shown by Cavalieri and Agarwal [48]. The wavenumbers respecting this inequality have been referred to as "acoustically matched" $[34,53]$. The radiating region of the spectrum is illustrated in figure 9 by the square delimited by the acoustic wavenumber. One can observe that, for the source with no coherence decay, all of the significant energy of the source is contained outside of the radiating region (figure 9a). We thus understand the significant difference in the acoustic efficiency. The effect of coherence decay is to stretch the source in wavenumber space, such that a more significant part of its energy is contained in the radiating part of the spectrum (figure $9 \mathrm{~b}$ ).

Although the two-point model with Gaussian amplitude envelope produces acoustic results that are much closer to the data, there are some discrepancies in SPL and directivity with respect to the experimental data, specially at low $S t$. In what follows we explore the differences between sound radiation of the Gaussian and asymmetric wavepackets. Figure 10 compares the SPL generated by both models with the acoustic data.

We also show the sound radiation obtained by directly applying equation 2.15 on the linearised $q_{11}$ term directly computed from the LES data. These results (the black solid lines in figure 10) can be thought of as the best ones that may be attained by the model using the current approach, because they correspond to the fully-computed CSD matrix, without any modelling. We can see the agreement between the sound radiation computed with this method and the acoustic data is quite good up to $S t=0.5$. Beyond that $S t$, overpredictions of approximately $5 \mathrm{~dB}$ are observed. This breakdown at higher $S t$ can be explained by the simplification made in equation 2.15 , in neglecting the radial structure of $J_{0}\left(k_{a} r \sin \theta\right)$. This term, named 'jet thickness' by Michalke [31] accounts for radial interferences of the sources within the jet, and its neglect yields an increase in sound radiation. As shown in table 1, neglecting the structure of the Bessel function starts to be problematic at low polar angles for $S t>0.5$; for instance, taking $r / D=2$ as the integration limit 


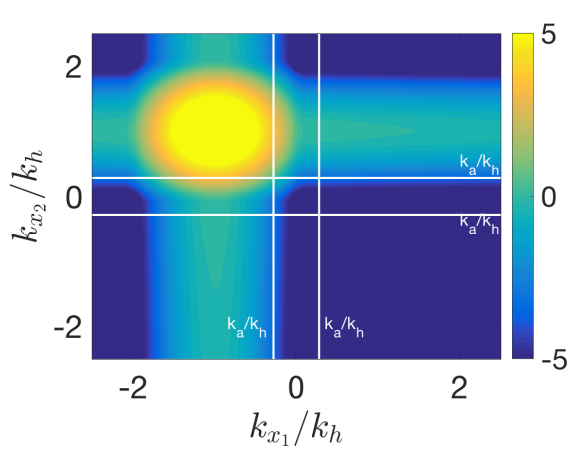

(a) $L_{c_{1,2}}=\infty$

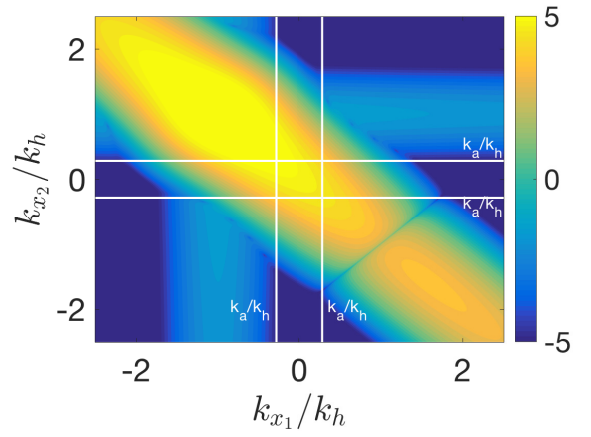

(b) $L_{c_{1,2}}=L_{c_{1,2, \text { num }}}$

Figure 9: Fourier transform of CSD in wavenumber space at $S t=0.3$. (a): source with unit coherence; (b): source with coherence modulation. Levels are in $\mathrm{dB}$ scale.

of equation 2.14 leads to an overestimation of approximately $20 \%$ in $J_{0}\left(k_{a} r \sin \theta\right)$ for low $\theta$. The results of the LES sound radiation depicted in figure 10 suggest that $S t=0.5$ is indeed the upper limit of the methodology we consider here.

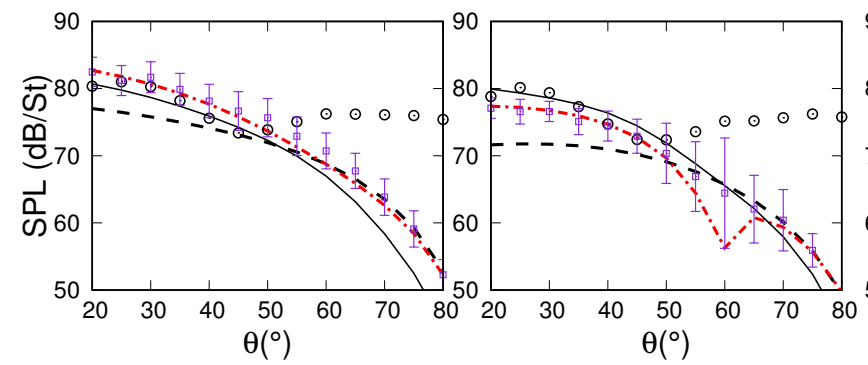

(a) $\mathrm{St}=0.3$ (b) $\mathrm{St}=0.4$

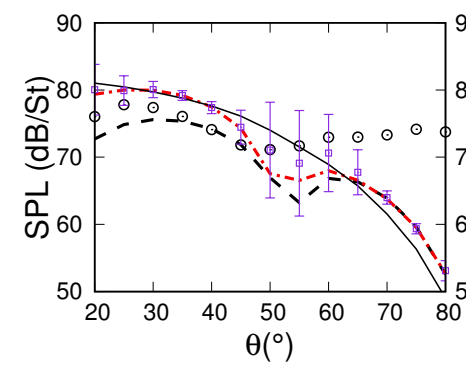

(d) $\mathrm{St}=0.6$

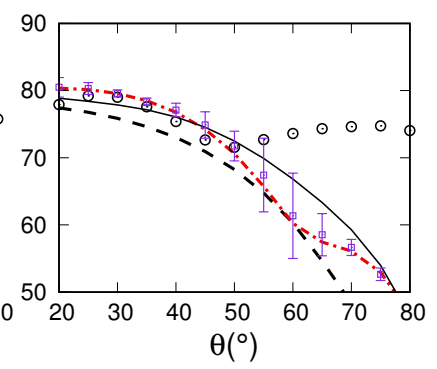

(c) $\mathrm{St}=0.5$

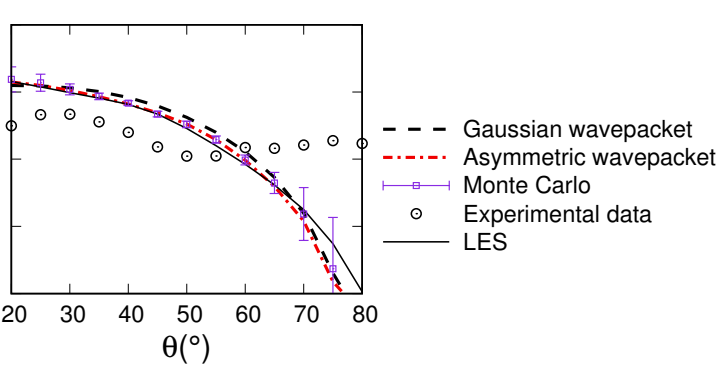

(e) $\mathrm{St}=0.7$

Figure 10: Sound radiation by sources with Gaussian an asymmetric amplitude envelopes. The error bars are centered at the mode of the sample values computed in the Monte Carlo simulation.

It may also be observed that the asymmetric wavepacket model provides better matches with the data, specially at lower $S t$, both in terms of amplitude and directivity. The sound radiation of the model is within $2 \mathrm{~dB}$ of the data to $\theta=50^{\circ}$. This reveals that a correct representation of the envelope is also a key aspect to be considered in order to make accurate acoustic predictions. 
As $S t$ is increased the difference between the two types of envelopes become less marked, as seen in figure 4, and the results of the two models become closer. At high polar angles, the agreement breaks down due to the simplifications made in the derivation of the model but also due to the lack of the other terms of the Lighthill stress tensor. As a matter of fact, one should expect the importance of the $q_{11}$ term to be reduced with increasing polar angle due to the $\cos ^{4} \theta$ directivity factor in equation 2.21. Modelling of these terms, which is beyond the scope of the work presented, is something to be considered for future work.

In order to assess the robustness of the model regarding its parameters, a Monte Carlo simulation was carried out for the model with the asymmetric envelope. We assumed that the parameters could vary at the same time by $\pm 10 \%$ about the values educed from the LES and that they followed a normal probability distribution; then the sound field was computed for 1000 random combinations of the parameters. The results of the Monte Carlo simulation can also be seen in figure 10, which shows the error bars centered around the most frequent value found in the simulation. It can be seen that the results of the asymmetric model are well within the range delimited by the error bars and are close to the most frequent value of the sample values computed. The maximum error found was approximately $3 \mathrm{~dB}$ up to $\theta=50^{\circ}$, and it increases in the polar range at which the model is no longer suitable for making acoustic predictions. These results reveal a certain robustness and the capacity of making accurate acoustic predictions even if numerical errors were to introduce errors in the eduction of the parameters.

The results of the kinematic models identify the parameters that dynamic models should be crafted to correctly predict. The phase speed is theoretically available from linear stability models. But the decay rate of the amplitude envelope, the coherence decay and the global amplitude are not readily available from such calculations. These parameters can only be determined by including an inhomogeneous term in the linearised equations, in which non-linear forcing will fix amplitude and determine the decay rate and coherence decay. In this framework, the resolvent of the linear operator is a key element, that determines how non-linear interactions force the linear waves. Studies $[73,74]$ have shown that there exists a close link between the optimal forcing and response modes of the resolvent operator and SPOD modes of wavepacket CSD. We keep this in mind in the following section where we perform SPOD of the source CSD with the asymmetric envelope (the one that gives more accurate acoustic predictions) with a view to exploring the possibility of low-rank modelling of the key source parameters highlighted by the results presented up to this point. Hereafter we shall concentrate our analysis up to $S t=0.5$, in the range where our methodology has been shown to provide accurate sound predictions.

\section{Spectral Proper Orthogonal Decomposition}

\section{(a) Equations}

The Proper Orthogonal Decomposition seeks a set of linear independent functions constituting a basis that best represents a given dataset in terms of fluctuation energy [87, 88]. For a turbulent flow, the POD functions are found through the maximisation of the mean-square projection on the velocity field, which is performed via the integral Fredholm equation, as shown by Lumley [87]

$$
\int S_{\hat{u} \hat{u}}\left(x_{1}, x_{2}\right) u_{\omega}\left(x_{2}\right) \mathrm{d} x_{2}=\sigma u_{\omega}\left(x_{1}\right) .
$$

In the case of SPOD, the kernel $S_{\hat{u} \hat{u}}$ is the cross-spectral density matrix of the field of interest, which is Hermitian by construction. The Fredholm equation can then be recast as an equivalent eigenvalue decomposition:

$$
S_{\hat{u} \hat{u}}=U \Sigma U^{-1} \text {. }
$$

Since $S_{\hat{u} \hat{u}}$ is Hermitian, $\mathrm{U}$ is a unitary matrix, so that $U^{H} U=I$ and $U^{H}=U^{-1}$. The eingenmode decomposition may then be rewritten as, 


$$
S_{\hat{u} \hat{u}}=U \Sigma U^{H},
$$

which corresponds to the singular value decomposition of $S_{\hat{u} \hat{u}}$.

Since the eigenvectors are orthogonal to each other, it is possible to construct a rank-N representation of the CSD as

$$
\widetilde{S}_{\hat{u} \hat{u}}=\sum_{k=1}^{N} u_{k} \sigma_{k} u_{k}^{H},
$$

where $\sigma_{k}$ are the eigenvalues and $u_{k}$ the eigenvectors of $S_{\hat{u} \hat{u}}$.

The elements of the rank- $\mathrm{N}$ representation of the CSD are given as,

$$
\left[\widetilde{S}_{\hat{u} \hat{u}}\right]_{x_{1}, x_{2}}=\sum_{k=1}^{N} u_{k}\left(x_{1}\right) \sigma_{k} u_{k}^{H}\left(x_{2}\right) .
$$

The rank-N representation of the coherence function is given as the rank-N CSD normalized by the rank-N PSD's at each of the points

$$
\widetilde{\gamma}^{2}\left(x_{1}, x_{2}\right)=\frac{\left|\sum_{k=1}^{N} u_{k}\left(x_{1}\right) \sigma_{k} u_{k}^{H}\left(x_{2}\right)\right|^{2}}{\left[\sum_{k=1}^{N} u_{k}\left(x_{1}\right) \sigma_{k} u_{k}^{H}\left(x_{1}\right)\right]\left[\sum_{k=1}^{N} u_{k}\left(x_{2}\right) \sigma_{k} u_{k}^{H}\left(x_{2}\right)\right]} .
$$

We can obtain the rank-1 coherence as,

$$
\widetilde{\gamma}^{2}\left(x_{1}, x_{2}\right)=\frac{\left|u_{1}\left(x_{1}\right) \sigma_{1} u_{1}^{H}\left(x_{2}\right)\right|^{2}}{\left[u_{1}\left(x_{1}\right) \sigma_{1} u_{1}^{H}\left(x_{1}\right)\right]\left[u_{1}\left(x_{2}\right) \sigma_{1} u_{1}^{H}\left(x_{2}\right)\right]}=\frac{\left|u_{1}\left(x_{1}\right)\right|^{2} \sigma_{1}^{2}\left|u_{1}\left(x_{2}\right)\right|^{2}}{\left|u_{1}\left(x_{1}\right)\right|^{2} \sigma_{1}^{2}\left|u_{1}\left(x_{2}\right)\right|^{2}}=1,
$$

showing that any single-mode representation of the CSD leads to unit coherence. In order to obtain two-point coherences lower than one, a superposition of $N>1$ modes is necessary.

The far-field radiation of a rank-N CSD may be assessed via equation 2.21 by substituting the source term with the low-order representation,

$$
\tilde{p}(R, \theta, \omega) \tilde{p}^{*}(R, \theta, \omega)=\frac{k_{a}^{4} \cos ^{4} \theta}{4 R^{2}} \iint\left[\widetilde{S}_{\hat{u} \hat{u}}\right]_{x_{1}, x_{2}} e^{i k_{a} \cos \theta\left(x_{1}-x_{2}\right)} \mathrm{d} x_{1} \mathrm{~d} x_{2} .
$$

\section{(b) Envelopes and phases}

SPOD was performed on the CSD issuing from both the LES data and the model. For the sake of compactness, throughout the remainder of the paper we shall concentrate on results of the SPOD carried out at $S t=0.3$ and $S t=0.5$. However, results for $S t=0.4$ have similar behaviour for all the plots in this and in the subsequent sections. Figure 11 shows the amplitude envelopes of the first four modes. The amplitudes have been normalised in order to allow qualitative comparisons between the modes of the two CSD's. The first mode is a typical wavepacket with a nearly gaussian envelope and subsequent modes have envelopes with successively larger number of lobes; for instance, $k^{t h}$ mode has $k$ lobes and $k-1$ minima points; this reflects the orthogonality of the basis that is formed by the modes. The spatial structure of the modes of the model CSD are in good agreement with those of the CSD issuing from the LES, especially for the first three modes. As the order of the model is increased, the tendency is that the agreement deteriorates, because a higher spatial resolution is required for the modes to be statistically converged [89].

The phases of the modes are shown in Figure 12. The phase of the first mode displays a typical convective behaviour characterised by a straight line. The higher-order modes have $\pi$ phase jumps at axial positions corresponding to the minima positions of the envelopes; these characteristics stem from the orthogonality of the basis formed by the eigenfunctions. While they cannot be directly related to any specific flow structures, one can interpret the higher-order SPOD 


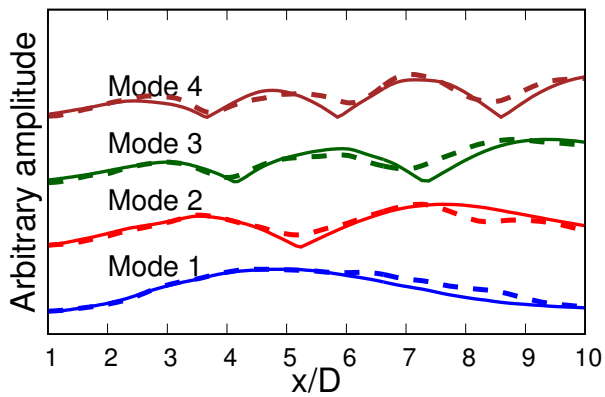

(a) $\mathrm{St}=0.3$

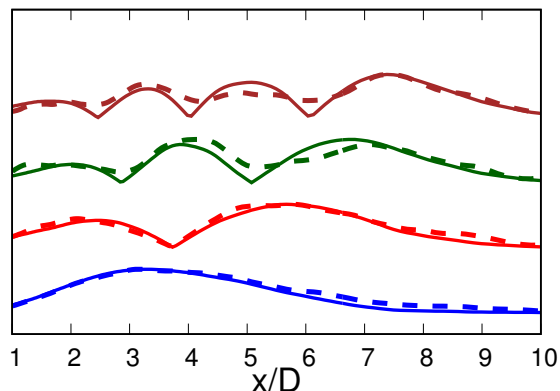

(b) $\mathrm{St}=0.5$

Figure 11: Structure of first 4 SPOD modes. Solid line represent the modes of the model CSD and dashed lines the modes of the CSD issued from the LES. The amplitudes are arbitrary and are in linear scale.

modes and their features as directions necessary to describe flow structures with more complex, jittery behaviour; therefore, they play a role in coherence decay by introducing a phase 'blur' between different axial stations. The phases of the modes of LES data also contain similar phase jumps.

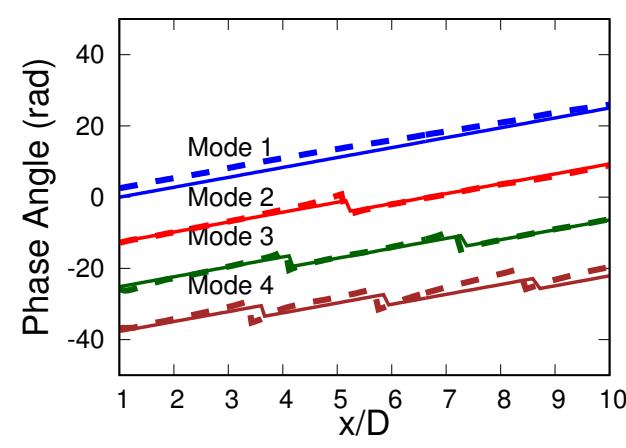

(a) $\mathrm{St}=0.3$

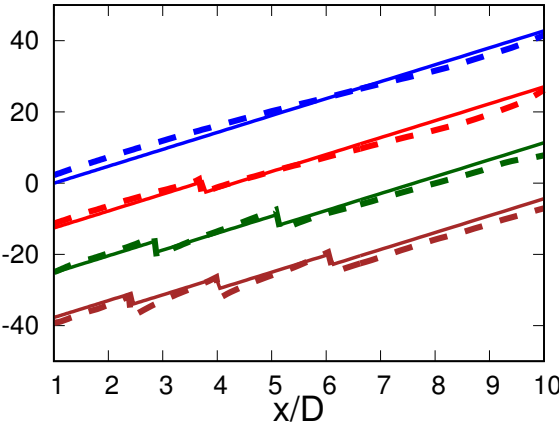

(b) $\mathrm{St}=0.5$

Figure 12: Phase of first 4 SPOD modes. Solid line represents the phases of the model CSD and dashed lines the phases of the CSD issued from LES data.

Furthermore, we can see from figure 11 that there is a certain characteristic lengthscale that can be associated with each mode by taking the distance between successive lobes; this lengthscale decreases with increasing mode number, suggesting that higher-order modes are associated with smaller scale activity.

As discussed in the introduction, the link between SPOD modes and resolvent modes has been explored by other studies [73-75], and the results indicate high correlations between leading SPOD and resolvent modes, suggesting that coherence decay is underpinned by a rather disorganised, uncorrelated background turbulence forcing the linearised equations of motion, and that the SPOD modes are structures that arise in response to this forcing and whose organisation is imposed by the linear operator. The envelopes and phases of the modes shown in Figures 11 and 12 suggest that background forcing leads to the multi-lobed structure and 
phase jumps manifest in the SPOD modes, which describe acoustically-important degrees of wavepacket freedom and that cannot be captured using a standard linear stability analysis that neglects the forcing due to nonlinearities. We now address the question of the rank necessary to describe these acoustically important dynamics, by computing sound fields using truncated CSD source models, and by evaluating the effect of truncation both on the radiated sound and the source parameters that underpin this.

\section{(c) Energy convergence}

The convergence of the SPOD regarding the energy of the modes may be evaluated through

$$
\frac{\sum_{i=1}^{k} \sigma_{i}}{\sum_{i=1}^{N} \sigma_{i}},
$$

where the sum of the energy captured by the first $k$ eigenvalues is normalised by the sum of the total energy. Figure 13 shows the energy convergence for the model CSD for $0.3 \leq S t \leq 0.5$. Between 10 and 20 modes are required to recover the energy of the full CSD, and it can be observed that the number of modes required to capture all of the energy contained in the CSD decreases as the Strouhal number is increased. This might lead to the conclusion that a low-rank behaviour only becomes pronounced with increasing Strouhal number. However, as can be seen in Figure 9, most of the energy of the source lies outside the radiating region in wavenumber space, so that the number of modes required to recover the energy is not necessarily the same to capture the important acoustic features of the CSD. Thus, in the next section we analyse the low-rank behaviour of the model regarding its acoustic radiation.

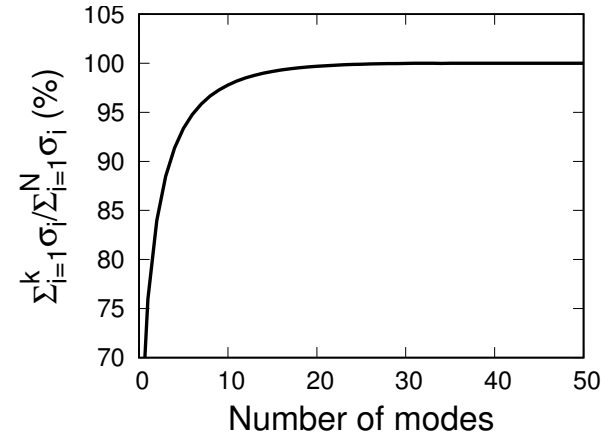

(a) $\mathrm{St}=0.3$

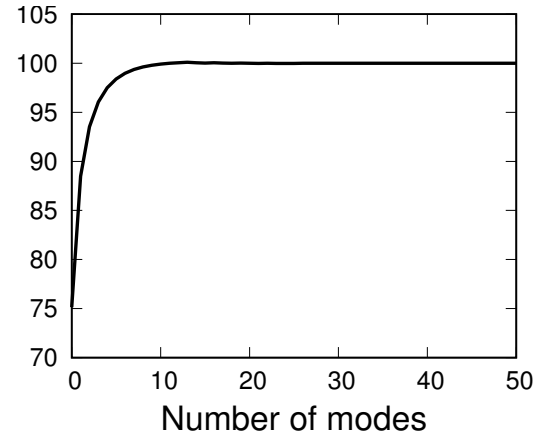

(b) $\mathrm{St}=0.5$

Figure 13: Cumulative energy of the first $k$ eigenvalues normalised by the sum of the $N$ computed eigenvalues.

\section{(d) Low-rank models}

We assess the convergence of low-rank approximations of the CSD of the kinematic source model with respect to acoustic radiation, and to two sound-source parameters considered earlier: amplitude envelope and coherence decay, which are not correctly captured by linear stability models.

Figure 14 shows the convergence of the envelopes of low-rank models with increasing numbers of modes with respect to the full CSD. We see that a one-mode representation 
underestimates the peak amplitude by approximately $20 \%$; nevertheless, we note that the lowrank models quickly converge to the amplitude envelope of the full CSD. Only 7 to 10 modes the reduced-order model suffice to capture the envelope length and amplitude.

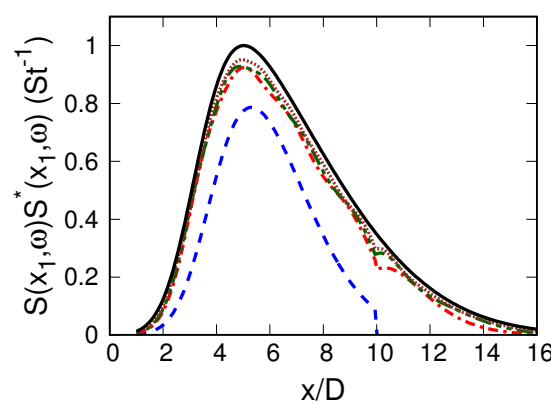

(a) $\mathrm{St}=0.3$

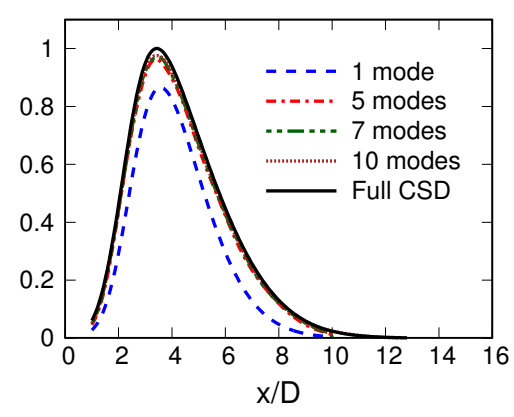

(b) $\mathrm{St}=0.5$

Figure 14: Wavepacket envelope convergence of different low-rank approximations. The levels have been normalised by the maximum value of the full source.

As shown in equation 6.7 equation, a low-rank representation of the CSD with only one mode results in unit coherence and so a higher-rank model is essential for this aspect of the wavepacket source behaviour. This is illustrated in Figure 15, which shows the coherence envelopes of lowrank approximations with up to 10 modes, with reference point at the center of the wavepacket, $x_{0}$. By successively adding more modes the coherence decays more sharply and approaches the envelope of the full CSD.

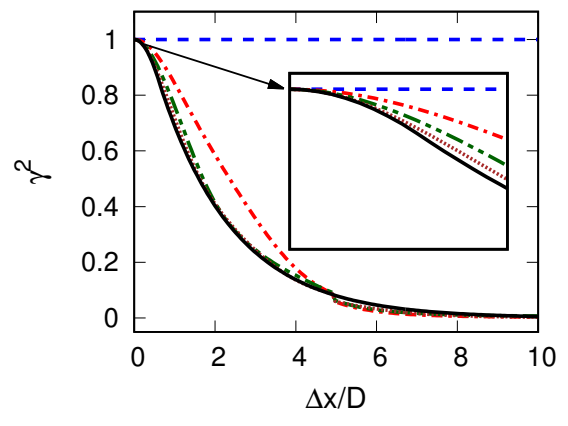

(c) $\mathrm{St}=0.3$

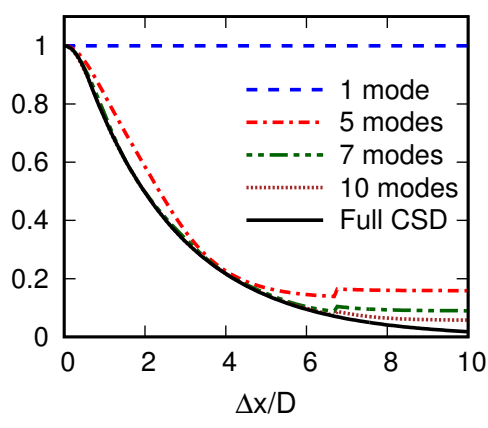

(b) $\mathrm{St}=0.5$

Figure 15: Coherence convergence of different low-rank approximations. Reference point is at $x_{0}$.

The SPL of a low-rank approximation can be computed using equation 6.8. We note that the number of modes needed to recover the acoustic radiation of the full CSD increases with increasing Strouhal number. Nevertheless, for the Strouhal numbers considered, a low-rank representation of the source with 10 modes was sufficient to recover the acoustic efficiency of the full source model at low polar angles, as seen in Figure 16. Due to its unit coherence, a lowrank model with only one mode has a very low acoustic efficiency, similar to the single-point wavepacket, as one can see by comparing Figures 8 and 16 . 


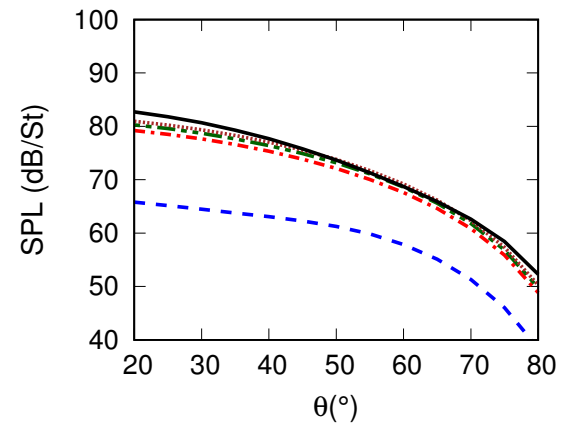

(a) $\mathrm{St}=0.3$

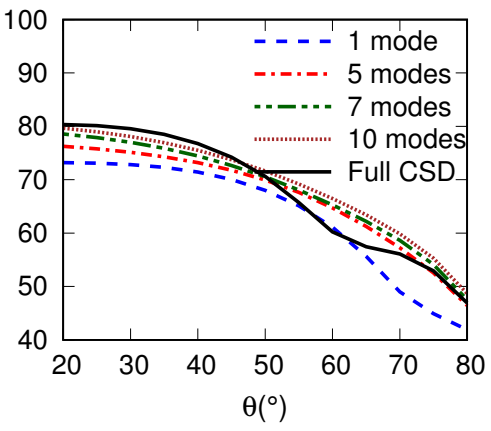

(b) $\mathrm{St}=0.5$

Figure 16: Effect of cumulative SPOD modes on far-field radiation by the wavepacket source.

10 modes is less than the number of modes necessary to recover all of the energy contained in the CSD (Figure 13) at lower Strouhal numbers $(S t \leqslant 0.3)$. This suggests that, at these Strouhal numbers, part of the energy is redundant from an acoustic point of view, and the correct representation of the wavepacket parameters is more relevant to sound radiation. These results reveal that this model source, which has been shown to produce the correct sound field at low polar angles, is relatively low-rank; wavepacket traits relevant to sound radiation, namely, coherence decay and envelope characteristic lengths, can be recovered with only a few SPOD modes.

\section{Conclusions}

The kinematic-sound source model proposed by Cavalieri and Agarwal [48] based on two-point statistics has been studied in order to get further insight into wavepacket parameters important to sound radiation. Data from a large-eddy simulation performed using the flow solver 'Charles' [85] was used to educe the physical parameters necessary to compute the sound radiation by the model source, namely: the wavepacket characteristic lengthscales, $L$ and $L_{a}$, the position where the envelope reaches its maximum, $x_{0}$, the envelope decay rate parameter, $\alpha$, the hydrodynamic wavenumber, $k_{h}$, and the coherence length scales, $L_{c_{1,2}}$.

Unlike past studies based on a line-source approach $[44,51,58,80]$, we choose to take account of the radial structure of the source. And unlike Cavalieri et al. [44], we educe the source parameters directly from the two-point velocity measurements instead of estimating single-point parameters from the sound field, following the approach of previous studies [11, 12, 21-24]. The single-point model does not account for coherence decay, and thus the single-point parameters inferred from the acoustic field do not correspond to those educed from flow data, as shown in Table 2. Due to the lack of coherence modulation in the single-point formulation, the correct source parameters can be considered to have been hidden in, or compensated for by, those inferred from the sound field; this highlights the caution that is necessary when using inverse approaches, as different source fields can lead to quite similar acoustic radiation. Using the parameters educed from LES data to animate the model, it was possible to compute the acoustic field through equation 2.21. The methodology presented here requires full-field data necessary to characterise the three-dimensional structure of the CSD matrix, which is at the core of Lighthill's acoustic analogy. This data can be obtained for instance, through a high-fidelity simulation with converged two-point statistics, as the one we use; or alternatively, it can be obtained from an experiment involving simultaneous measurements in two-different flow planes, as the one carried out by Jaunet et al. [19]. In the absence of full-field data, simplifying assumptions about the 
structure of the CSD matrix have to be done, which can lead to the use of empirical constants to calibrate the source.

The results reveal the key importance of both coherence decay and envelope shape for wavepacket sound radiation. They show that, if coherence decay is neglected, most of the energy of the model source lies outside of region of the spectrum corresponding to acoustically-matched wavenumbers, and thus the single-point wavepacket has a very low acoustic efficiency. These results highlight the importance of coherence decay, the frequency-domain manifestation of jitter, for jet-noise modelling, which has been evoked in previous studies [41, 47, 51, 52].

Furthermore, it has been shown that the asymmetry of the wavepacket envelope is also of key importance for an accurate sound prediction. An asymmetric envelope, which better represents flow data, produces sound levels closer to experimental results at low polar angles, especially at low Strouhal numbers. For $S t>0.5$ the model overpredicts the data due to the acoustic compactness assumption, which amounts to neglecting the radial structure of the Bessel function, $J_{0}\left(k_{a} r \sin \theta\right)$, in the far field radiation equation. These observations give insight regarding perspectives for dynamic wavepacket models to predict jet noise: although the standard, unforced linear models successfully capture the phase speed and the amplitude envelope up to the point where the Kelvin-Helmholtz mode becomes stable [35, 40, 41], they underpredict the amplitude further downstream and do not contain information regarding coherence decay, making an accurate noise prediction impossible; on the other hand, other studies have shown that these issues may be overcome by use a forcing term in the linearised equations [90]. This and the fact that forced linear stability models can determine the amplitude of the fluctuations make them more suitable to describe sound generation mechanisms.

A Spectral Proper Orthogonal Decomposition of the model CSD was performed with a view to exploring the possibility of constructing a reduced-rank source model capable of capturing the acoustically-important parameters. This is further motivated by the recently demonstrated link between SPOD modes and optimal resolvent response modes that arise when the linear stability models are considered in the presence of stochastic forcing [75].

An analysis of eigenvalue convergence reveals that approximately 10 to 20 modes are necessary to capture the total energy of the CSD, the number of modes decreasing with increasing Strouhal number. On the other hand, only 10 modes are necessary to recover the most important traits for sound radiation, namely the envelope shape and coherence envelopes. This reveals that the acoustically-important features can be reproduced with a low-rank model.

\section{Data accessibility}

The numerical and acoustic data, as well as the codes used to generate the results presented in this work are accessible through the figshare repository at: https://figshare.com/s/ 73ba8c11f4ed4183d669.

\section{Competing Interests}

The authors have no competing interests.

\section{Authors' contributions}

I.A.M treated the LES data in order to extract the wavepacket parameters and implemented the mathematical model and the codes used to compute the acoustic radiation of the model and the SPOD analysis. I.A.M, P.J and A.V.G.C derived and revised the mathematical model. V.J contributed to the acoustic experiments and to the post-processing of the LES data and also participated in the coding and discussions concerning the SPOD. All authors worked on the analysis and interpretation of the results and on the writing of the manuscript. 


\section{Acknowledgements}

The authors wish to acknowledge Selene Piantanida for her work during the acoustic measurements at Bruit et Vent. The authors would also like to thank Dr. Guillaume Brès at Cascade Technologies for providing the simulation database. The LES work was supported by NAVAIR SBIR project under the supervision of J. T. Spyropoulos, with computational resources provided by DoD HPCMP.

\section{Funding statement}

This work was supported by CNPq - National Council of Scientific and Technological Development - Brazil through the Science Without Borders program under grant number 200676/2015-6, and also through project A073/2013.

\section{References}

1 M. J. Lighthill. On sound generated aerodynamically i. general theory. In Proceedings of the Royal Society of London A: Mathematical, Physical and Engineering Sciences, volume 211, pages 564-587. The Royal Society, 1952.

2 G. M. Lilley. On the noise from jets. In AGARD Conference proceedings, number 131, 1974.

3 M. E. Goldstein. A generalized acoustic analogy. Journal of fluid mechanics, 488:315-333, 2010.

4 S.C. Crow. Aerodynamic sound emission as a singular perturbation problem. Studies in Applied mathematics, 1970.

5 J. B. Freund. Noise-source turbulence statistics and the noise from a mach 0.9 jet. Physics of Fluids, 15(6), 2003.

6 T. Colonius and J. B. Freund. Application of lighthill's equation to a mach 1.92 turbulent jet. AIAA Journal, 368, 2000.

7 P. O. A. L. Davies, M. J. Fisher, and M. J. Barrat. The characteristics of turbulence in the mixing region of a round jet. Journal of Fluid Mechanics, 15:337-367, 1963.

8 P. Bradshaw, D. H. Ferris, and R. F. Johnson. Turbulence in the noise-producing region of a circular jet. Journal of Fluid Mechanics, 19:591-624, 1964.

9 P. O. A. L. Davies. Turbulence structure in freee-shear layers. AIAA journal, 4:1971-1978, 1966.

10 W. T. Chu. Turbulence measurements relevant to jet noise. Technical Report 19, UTIAS, University of Toronto, Toronto, Canada, November 1966.

11 M. Harper-Bourne. Jet near-field noise prediction. In Proceedings of the 5th AIAA/CEAS Aeroacoustics Conference and Exhibit, number 1838, 1999.

12 M. Harper-Bourne. Jet noise turbulence measurements. In Proceedings of the 9th AIAA/CEAS Aeroacoustics Conference and Exhibit, number 3214, 2003.

13 P. J. Morris and K. B. M. Q. Zaman. Velocity measurements in jets with application to noise source modelling. Journal of Sound and Vibration, 239:394-414, 2010.

14 P. Jordan and Y. Gervais. Modelling self- and shear-noise mechanisms in inhomogeneous, anisotropic turbulence. Journal of Sound and Vibration, 279:529-555, 2005.

15 F. Kerhervé, J. Fitzpatrick, and P. Jordan. The frequency dependency of jet turbulence for noise source modelling. Journal of Sound and Vibration, 296:209-225, 2006.

16 L. Ukeiley, C. Tinney, R. Mann, and M. Glauser. Spatial correlations in a transonic jet. AIAA journal, 45(6):1357-1369, 2007.

17 J. M. Seiner, L. Ukeiley, and M. K. Ponton. Jet noise source measurements using piv. In Proceedings of the 5th AIAA/CEAS Aeroacoustics Conference and Exhibit, number 1869, 1999.

$18 \mathrm{~J}$. Bridges and M. Wernet. Measurements of the aeroacoustic sound source in hot jets. In Proceedings of the 10th AIAA/CEAS Aeroacoustics Conference and Exhibit, number 212508, 2004.

19 V. Jaunet, P. Jordan, and A. V. G. Cavalieri. Two-point coherence of wavepackets in turbulent jets. Physical Review Fluids, 2(024604), 2017.

20 S. A. Karabasov, M. Z. Afsar, T. P. Hynes, A. P. Dowling, W. A. MacMullan, C. D. Pokora, G. J. Page, and J. J. McGuirk. Jet noise: acoustic analogy informed by large eddy silulation. AIAA journal, 48(7), July 2010. 
21 R. H. Self. Jet noise prediction using lighthill acoustic analogy. Journal of Sound and Vibration, 275:757-768, 2004.

22 M. E. Goldstein and A. Kharavan. Acoustic source modelling for high speed air jets. In Proccedings of the 43rd Aerospace Sciences Meeting and Exhibit, volume 213416, Reno, Nevada, January 2005.

23 M. E. Goldstein and S. J. Leib. The aeroacoustics of slowly diverging supersonic jets. Journal of fluid mechanics, 600:291-337, 2008.

24 S. J. Leib and M. E. Goldstein. Hybrid source model for predicting high-speed jet noise. AIAA journal, 49(7):1324-1335, July 2011.

25 C. K. W. Tam and L. Auriault. Jet mixing noise from fine-scale turbulence. AIAA journal, 37(2): 145-153, 1999.

26 A. Kharavan, E. A. Z. Krejsa, and C. M. Kim. Computation of supersonic jet mixing noise for an axisymmetric convergent-divergent nozzle. Journal of aircraft, 31(3):603-609, 1994.

27 E. Mollo-Christensen. Measurements of near field pressure of subsonic jets. Technical report, DTCI, 1963.

28 E. Mollo-Christensen. Jet noise and shear flow instability seen from an experimenter's viewpoint. Journal of Applied Mechanics, 34:1-7, 1967.

29 S. Crow and F. Champagne. Orderly structure in jet turbulence. Journal of Fluid Mechanics, 48 547-591, 1971.

30 A. V. G. Cavalieri, P. Jordan, and L. Lesshafft. Wave-packet models for jet dynamics and sound radiation. Applied Mechanics Reviews, 2019.

31 A. Michalke. A wave model for sound generation from circular jets. Technical report, Deutsche Luft- und Raumfart, 1970.

32 A. Michalke. An expansion scheme for the noise from circular jets. Zeitschrift für Flugwissenschaften, 20:229-237, 1972.

33 D. G. Crighton and M. Gaster. Stability of slowly diverging jet flow. Journal of Fluid Mechanics, 77:397-413, 1976.

34 P. Jordan and T. Colonius. Wave packets and turbulent jet noise. Annual Review of Fluid Mechanics, 45:173-195, 2013.

35 A. V. G. Cavalieri, D. Rodríguez, P. Jordan, T. Colonius, and Y. Gervais. Wavepackets in the velocity field of turbulent jets. Journal of fluid mechanics, 730:559-592, 2013.

36 F. Kerhervé, P. Jordan, A. V. G. Cavalieri, J. Delville, C. Bogey, and D. Juvé. Educing the source mechanism associated with downstream radiation in subsonic jets. Journal of Fluid Mechanics, 710:606-640, 2012.

37 D. Rodríguez, A. V. G. Cavalieri, T. Colonius, and P. Jordan. A study of wavepacket models for subsonic turbulent jets using local eingemode decompostion of piv data. European Journal of Mechanics B/Fluids, 49:308-321, 2015.

38 C. E. Tinney and P. Jordan. The near pressure field of co-axial subsonic jets. Journal of Fluid Mechanics, 611:175-204, 2008.

39 T. Suzuki and T. Colonius. Instability waves in a subsonic round jet detected using a near-field phased microphone array. Journal of Fluid Mechanics, 565:197-226, 2006.

$40 \mathrm{~K}$. Gudmundsson and T. Colonius. Instability wave models for the near-field fluctuations of turbulent jets. Journal of Fluid Mechanics, 689:97-128, 2011.

41 David E. S. Breakey, Peter Jordan, André V. G. Cavalieri, Petrônio A. Nogueira, Olivier Léon, Tim Colonius, and Daniel Rodríguez. Experimental study of turbulent-jet wave packets and their acoustic efficiency. Phys. Rev. Fluids, 2:124601, 2017.

42 S. C. Crow. Acoustic gain of a turbulent jet. In Paper IE.6, Meeting of Division of Fluid Dynamics, American Physical Society, Boulder, November 1972. University of Colorado.

43 A. Michalke and H. V. Fuchs. On turbulence and noise of an axisymmetric shear flow. Journal of Fluid Mechanics, 70:179-205, 1975.

44 A. V. G. Cavalieri, P. Jordan, T. Colonius, and Y. Gervais. Axisymmetric superdirectivity in subsonic jets. Journal of Fluid Mechanics, 704:338-420, 2012.

45 V. Kopiev, S. Chernyshev, G. Faranosov, M. Zaitsev, and I. Belyaev. Correlations of jet noise azimuthal components and their role in source identification. In 16th AIAA/CEAS Aerocoustics Conference, Stockholm, 2010. AIAA. 
46 G. Brès A., P. Jordan, V. Jaunet, M. Le Rallic, A. V. G. Cavalieri, A. Towne, S. K. Lele, T. Colonius, and O. T. Schmidt. Importance of the nozzle-exit boundary-layer state in subsonic turbulent jets. Journal of Fluid Mechanics, 851:83-124, 2018.

47 Y. B. Baqui, A. Agarwal, A. V. G. Cavalieri, and S. Sinayoko. A coherence-matched linear mechanism for subsonic jets. Journal of Fluid Mechanics, 776(235-267), 2015.

48 A. V. G. Cavalieri and A. Agarwal. Coherence decay and its impact on sound radiation by wavepackets. Journal of Fluid Mechanics, 748:399-415, 2014.

49 P. Jordan, T. Colonius, G. A. Brès, M. Zhang, A. Towne, and S. Lele. Modelling intermittent wavepackets and their radiated sound in a turbulent jet. In Summer Program. Center for Turbulent Research, 2014.

50 M. Zhang, P. Jordan, G. Lehnasch, A. V. G. Cavalieri, and A. Agarwal. Just enough jittering for jet noise. In Proceedings of the 19th AIAA/CEAS Aeroacoustics Conference and Exhibit, Atlanta, GA, Unites States, 2014. AIAA.

51 A. V. G. Cavalieri, P. Jordan, A. Agarwal, and Y. Gervais. Jittering wave-packet models for subsonic jet noise. Journal of Sound and Vibration, 330:4474-4492, 2011.

52 J. E. Ffwocs-Williams and A. J. Kempton. The noise from the large-scale structure of a jet. Journal of Fluid Mechanics, 84:673-694, 1978.

53 D. G. Crighton. Basic principles of aerodynamic noise generation. Progress in aerospace science, 16(1):31-96, 1975.

54 D. G. Crighton and P. Huerre. Shear-layer pressure fluctuations and superdirective acoustic sources. Journal of Fluid Mechanics, 220:355-368, 1990.

55 A. V. G. Cavalieri, P. Jordan, W. R. Wolf, and Y. Gervais. Scattering of wavepackets by a flat plate in the vicinity of a turbulent jet. Journal of Sound and Vibration, 333:6516-6531, 2014.

56 S. Piantanida, V. Jaunet, J. Huber, W. R. Wolf, P. Jordan, and A. V. G. Cavalieri. Scattering of turbulent-jet wavepackets by a swept trailing edge. The Journal of the Acoustical Society of America, 140:4350-4359, 2016.

57 P. A. S. Nogueira, A. V. G. Cavalieri, and P. Jordan. A model problem for sound radiation by and installed jet. In Proceedings of the 22th AIAA/CEAS Aeroacoustics Conference and Exhibit, Lyon, France, June 2016. AIAA.

58 F. D. da Silva, C. J. Deschamps, P. Jordan, S. Piantanida, A. V. G. Cavalieri, and G. Brès. Effects of coherence on jet-surface interaction noise. In Proceedings of the 22th AIAA/CEAS Aeroacoustics Conference and Exhibit, Lyon, France, June 2016. AIAA.

59 A. Michalke. Some remarks on source coherence affecting jet noise. Journal fo sound and vibration, 87:1-17, 1983.

60 R. Reba, S. Narayanan, T. Colonius, and T. Suzuki. Modeling jet noise from organized structures using near-field hydrodynamic pressure. In Proceedings of the 11th AIAA/CEAS Aeroacoustics Conference and Exhibit, number 3093, Monterey, California, United States, May 2005.

61 R. Reba, J. Simonich, and R. Schlinker. Measurements of source wave-packets in high-speed jets and connection to far-field sound. In Proceedings of the 14th AIAA/CEAS Aeroacoustics Conference and Exhibit, number 3256, Vancouver, British Columbia, Canada, May 2008.

62 R. Reba, J. Simonich, and R. Schlinker. Sound radiated by large-scale wave-packets in subsonic and supersonic jets. In Proceedings of the 15th AIAA/CEAS Aeroacoustics Conference and Exhibit, number 3256, Miami, Florida, United States, May 2009.

63 R. Reba, S. Narayanan, and T. Colonius. Wave-packet models for large-scale mixing noise. International journal of aeroacoustics, 9(4):533-558, 2010.

64 G. Brès, F. E. Ham, J. W. Nichols, and S. K. Lele. Unstructured alrge-eddy simulations of supersonic jets. AIAA journal, 55(4):1164-1184, 2017.

65 V. F. Kopiev and S. A. Chernyshev. Simulation of azimuthal characteristics of turbulent jet noise by correlation model of quadrupole sources. International Journal of Aeroacoustics, 13(1): 39-60, 2014.

66 V. F. Kopiev and S. A. Chernyshev. Refraction effect in correlation model of quadrupole noise sources in turbulent jet. In Proceedings of the 21st AIAA/CEAS Aeroacoustics Conference and Exhibit, number 3130, Dallas, United States, 2015. 
67 V. F. Kopiev and S. A. Chernyshev. Correlation model for noise sources in turbulent jet based on birth of vortices as a key event. In Proceedings of the 18th AIAA/CEAS Aeroacoustics Conference and Exhibit, number 2250, Colorado Springs, United States, 2012.

68 B. F. Farell and P. Ioannou. Accurate low-dimensional approximation of the linear dynamics of fluid flow. Journal of the Atmospheric Sciences, 58(18):2771-2789.

69 Y. Hwang and C. Cossu. Linear non-normal energy amplification of harmonic and stochastic forcing in the turbulent channel flow. Journal of Fluid Mechanics, 654:51-73, 2010.

70 S. Bagheri, D. S. Henningson, J. Hoepffner, and P. J. Schmid. Input-output analysis and control design applied to a linear model of spatially developing flows. Applied Mechanics Reviews, 62(2) (020803), 2009.

71 B. J. McKeon and A. S. Sharma. A critical-layer framework for turbulent pipe flow. Journal of Fluid Mechanics, 658:336-382, 2010.

72 G. Tissot, M. Zhang, F. C. Lajús Jr., A. V. G. Cavalieri, and P. Jordan. Sensitivity of wavepackets in jets to nonlinear effects: the role of the critical layer. Journal of Fluid Mechanics, 811:95-137, 2017.

73 O. Semeraro, V. Jaunet, P. Jordan, A. V. G. Cavalieri, and L. Lesshafft. Stochastic and harmonic optimal forcing in subsonic jets. In Proceedings of the 21st AIAA/CEAS Aeroacoustics Conference and Exhibit, Lyon, France, 2016. AIAA.

74 A. Towne, T. Colonius, P. Jordan, A. V. G. Cavalieri, and G. Brès. Stochastic and nonlinear forcing of wavepackets in a mach 0.9 jet. In Proceedings of the 21st AIAA/CEAS Aeroacoustics Conference and Exhibit, Dallas, United States, 2015. AIAA.

75 Aaron Towne, Oliver T. Schmidt, and Tim Colonius. Spectral proper orthogonal decomposition and its relationship to dynamic mode decomposition and resolvent analysis. Journal of Fluid Mechanics, 847:821-867, 2018.

76 T. Suzuki. Coherent noise sources of a subsonic round jet investigated usqing hydrodynamic and acoustic phased-micriphone arrays. Journal of fluid mechanics, 730:659-698, 2013.

77 M. T. Landahl and E. Mollo-Christensen. Turbulence and random processes in fluid mechanics. Cambridge University Press, 1992.

78 D. J. Bodony and S. K. Lele. Low-frequency sound sources in high-speed turbulent jets. Journal of Fluid Mechanics, 617:231-253, 2008.

79 M. Goldstein. Aeroacoustics. McGraw-Hill.

80 P. A. S. Nogueira, A. V. G. Cavalieri, and P. Jordan. A model problem for sound radiation by an installed jet. Journal of Sound and Vibration, 391:95-115, 2017.

81 P. Jordan, R. Wells, Y. Gervais, and J. Delville. Optimisation of correlation function models for statistica aeroacoustic noise prediction. In CFA/DAGA Aeroacoustics Conference, 2004.

82 D. O'Hara, N. Andersson, P. Jordan, M. Bilsson, L. Eriksson, and L. davidson. A hybrid analysis methodology for improved accuracy in low cost jet noise modelling. In 33rd International Congress and Exposition on Noise Control Engineering, San Francisco, 2004.

83 D. Papamouschou. Prediction of jet noise shielding. In Proceedings of the 48th AIAA Aerospace Sciences Meeting, 2010.

84 M. Koenig, A. V. G. Cavalieri, P. Jordan, J. Delville, and Y. Gervais. Farfield filtering and source imaging of subsonic jet noise. Journal of Sound and Vibration, 332(18):4067-4088, 2013.

85 O. T. Schmidt, A. Towne, G. Rigas, T. Colonius, and G. A. Brès. Spectral analysis of jet turbulence. Journal of Fluid Mechanics, 855:953-982, 2018.

86 V. F. Kopiev and S. A. Chernyshev. New correlation model for the cascade of turbulent pulsations as a noise source in turbulent jets. Acoustical Physics, 58(4):442-456, 2012.

87 J. L. Lumley. The structure of inhomogeneous turbulent flows. Atmospheric Turbulence and Radio Wave Propagation, pages 168-178, 1967.

88 J. H. Citriniti and W. K. George. Reconstruction of the global velocity field in the axisymmetric mixing layer utilizing the proper orthogonal decomposition. Journal of Fluid Mechanics, 418: 137-166, 2000.

89 V. Jaunet. Convergence of pod modes. Private communications, 2017.

90 P. Jordan, M. Zhang, G. Lehnasch, and A. V. G. Cavalieri. Modal and non-modal linear wavepacket dynamics in turbulent jets. In Proceedings of the 23rd AIAA/CEAS Aeroacoustics Conference, Denver, United States, June 2017. 